\title{
Do Older Employees Have a Lower Individual Productivity Potential than Younger Employees?
}

\author{
Pål Børing ${ }^{1}$ • Jens B. Grøgaard ${ }^{1,2}$
}

Received: 15 August 2020 / Accepted: 21 December 2020/Published online: 02 January 2021

(C) The Author(s) 2021

\begin{abstract}
We examine the relationship between employees' age and their individual productivity potential (IPP). IPP is measured by individual characteristics which are related to skills utilisation at work. Using PIAAC data for 27 European and non-European countries, we find that the oldest employees have a lower IPP score than the middle-aged employees in 17 of the 27 countries. It seems to be most demanding for the oldest workers to keep up with the IPP of younger workers in countries with high average skill loss for the oldest age group or high average skills level for all age groups (or both). The significant positive effects of formal education and the absence of significant effects of skills on the IPP score in many of the countries (the individual level), are easier to adapt to the human capital perspective on the importance of formal education than to the perspective of the signalling theory.
\end{abstract}

Keywords Individual productivity potential $\cdot$ Age groups $\cdot$ Employees $\cdot$ Human capital theory $\cdot$ Signalling theory $\cdot$ Labour contracts

\section{Introduction}

People live longer than ever before, and birth rates decline in the majority of OECD countries (OECD 2013). This leads to visible shifts in the age composition of the population. At the global level, the proportion of persons aged 60 years or older is predicted to increase from $12.3 \%$ in 2015 , and continue to increase to $21.5 \%$ by 2050 (United Nations 2015). In Europe as a whole, this level has already been reached, and is expected to increase further from $23.9 \%$ in 2015 to $34.2 \%$ by 2050 (ibid.). This

Pål Børing

paal.boring@nifu.no

1 NIFU (Nordic Institute for Studies in Innovation, Research and Education), Økernveien 9, N-0653 Oslo, Norway

2 School of Business, Department of Business, History and Social Sciences, University of South-Eastern Norway (USN), Campus Vestfold, N-3199 Borre, Norway 
development represents an array of financial and social challenges. In line with OECD's (2013) recommendations, it is important to increase the employability of older adults and their motivation to postpone retirement, in order to facilitate a more active ageing and thereby to reduce public costs for pensions and health care. Population ageing and related financial sustainability concerns are pushing policy makers to adopt an increase in the formal retirement age. But the major challenge is to increase the real retirement age for many workers. To achieve this, older employees with earned rights must be motivated to extend their professional careers and those who are out of work must be included again. This requires regulatory changes and facilitation from the state, but it also requires concrete follow-up from employers in the public and private sectors.

So, we may ask, how does the business community deal with such challenges? Conducted surveys indicate that policy-directed attempts to postpone claims for social security benefits and the retirement of older workers may be counteracted by age discrimination (Neumark and Song 2013; Carlsson and Eriksson 2019; Neumark 2020). The Norwegian Senior Policy Barometer of 2016 shows that $37 \%$ of managers do not consider inviting applicants for vacancies for interviews unless they have at least ten years left of their professional career. Exploring the possible effects of the financial crisis in Norway on managers' attitudes to older workers, Solem (2012) concludes that older workers are among the least popular categories to recruit in both rising and falling cycles. According to OECD (2013), 'individuals already in their early 50s may meet barriers in the labour market mainly because of their age' (p. 92). These are expressions of attitudes that respondents often justify with reference to their personal experience. Many managers suggest that older workers are associated with lower profitability for the firm. They assume that older workers have lower learning ability and adaptability than younger workers. Using surveys of employees and employers in the Netherlands, Van Dalen et al. (2010) find that both employers and employees rate the productivity of older workers substantially lower than that of younger workers. Barthel (2008) emphasises that it is a widespread stereotype that older workers are probably less productive: 'the arguments given in the literature are that the physical abilities decrease in general, cognitive abilities at least in some areas' (ibid., p. 3).

It may therefore be unrealistic to expect employers to change their recruitment strategy of re-entering older workers into the labour market due to rationalisation, restrictions, closures, etc. We now ask whether these attitudes towards older workers are also propagated in the workplace. Is it the case that the allocation of work tasks that are learning and developing, advanced and demanding is also negatively affected by the employee's age? To answer this question, we use the concept 'individual potential productivity' (IPP). Skirbekk (2004) outlines how human capital variables as mental and physical abilities, education and job experience form an individual's productivity potential. Skirbekk (2008) aims to provide estimates for a framework to measure productivity potential by age, where this measurement is based on cognitive and noncognitive skills and the labour market importance of these skills.

Based on this theory of IPP, we use PIAAC (Programme for the International Assessment of Adult Competencies) data from the OECD to construct a measure of activities that promote or enhance employees' IPP in the workplace. IPP is measured by five characteristics which are originally intended to measure the skills content of the respondent's job, and thus which may be regarded as proxies for the utilisation of skills 
at work, see OECD (2016, p. 96). Two of these characteristics are related to informal learning at work (learning new work-related things, and learning by-doing from tasks), a third one is related to innovative learning (keeping up to date with new products), and the two remaining characteristics concern instructing, training, teaching or advising people. Skirbekk $(2004,2008)$ argues that such job-related characteristics are among several factors that form an individual's productivity potential, but these aspects of job performance do not necessarily measure individual productivity directly, at best they are proxies for productivity at work. We contribute to this theory by examining how the allocation of such activities is related to the age of the employees, conditioned by selected human capital variables such as cognitive skills, formal education and the employee's profession and industrial affiliation. We also examine how these relationships appear in 27 European and non-European countries.

According to the OECD, PIAAC measures adults' proficiency in key information skills - literacy, numeracy and problem solving in a technically advanced (digital) environment - and gathers information on how adults use their skills at work, at home and in the wider community (OECD 2016). PIAAC has been conducted in three rounds covering more than 40 countries. Our dataset covers the two first rounds of the survey and contains 31 countries, of which 27 countries have a complete data set on the variables we analyse.

Economic theory usually operationalises productivity as the wage level, and PIAAC demonstrates that an increase in the literacy scale of one standard deviation is associated with an increase in the likelihood of being employed (versus unemployed) by 0.8 percentage point when formal education and socio-demographic characteristics of the employee are kept constant. Analogously, an increase of one standard deviation in literacy proficiency is associated with a $6 \%$ increase in the wage level, on average, ceteris paribus (OECD 2016, p. 17). The correlation (Pearson's r) between the level of formal education and literacy score is 0.523 (31 countries), which means that an increase in formal education level with one standard deviation is associated with an increase in skills level by approximately 0.5 standard deviation, on average (Grøgaard and Børing 2017, p. 26). But at the same time, the implication of this is that individual productivity is not captured by one and only one empirical indicator, e.g. the wage level of the employee. As emphasised in OECD (2013), experience and expertise are beneficial in some jobs, while physical strength and quick reactions are beneficial in others. Van Ours and Stoeldraijer (2011) add that individual productivity is multidimensional. They mention a number of characteristics that relate to productivity, ranging from human capital variables like cognitive skills and formal education to personal motivation and job experience. ${ }^{1}$

Productivity can also be measured at the firm level, but then it becomes problematic to separate out the personal contribution to the firm's production value. The OECD (2013) argues that this inter alia is due to contributions from other confounding factors and the general lack of precise data linking individual to joint or collective productivity. Wage levels are affected by both skills, level of education and socio-demographic characteristics, and relative wages are affected by the institutional settings in different

\footnotetext{
${ }^{1}$ Van Ours and Stoeldraijer (2011) list communication skills, information processing speed, strength and endurance, health, self-discipline, flexibility, administrative and strategic capacities, math proficiency, vocabulary size, education, motivation, energy and job experience.
} 
countries, e.g. by their wage-bargaining systems. Scandinavian countries have a more compressed wage structure (smaller wage differences) than most other OECD countries, but these countries have a highly productive export-oriented business sector which is at least as productive as comparable sectors in OECD countries with larger wage differences (Moene and Wallerstein 2003, 2005; Freeman 1997).

Most of the presented studies in this article which evaluate how age is related to productivity are based on individual- or firm-level data for a single country. We contribute to the research literature by examining whether there are country-specific differences in the relationship between age and IPP based on the individual-level PIAAC data. We also examine whether the estimation results can be explained on the basis of country-specific differences in the average IPP score, the average skill loss for the oldest age group, the average skills level, and the proportion of employees with higher education, at the national level. In this way, we contribute to the theory of IPP in the workplace, and we conduct an empirical study which in this context can be used to test what we perceive as key hypotheses in human capital theory and signalling theory. The analyses also provide information about the connection between employees' age and firms' allocation of what we perceive as interesting and learning work tasks in an employment relationship, and above all how such relationships vary between several countries.

We first analyse the PIAAC data using two models, M1 and M2. M1 (Table 2) reveals the bivariate relationship between the employee's age and the score on the IPP index in the 27 countries. M2 (Table 2) controls the relationship between age and IPP for formal education, cognitive skills, occupation, industrial affiliation and many other variables. Since age is the underlying variable, significant control variables act as potential mediators of age effects on IPP in M2. If the age effect is robust, i.e. it changes modestly from M1 to M2, significant control variables will operate as an explanatory addition to age in the IPP regressions. We also test whether changes in age effects are significant when controlling for 3rd variables (M3 and M4, Table 3). In addition, we conduct a multi-level analysis (M5 and M6, Table 4). These models are described in detail in "The Estimation Results" section.

The rest of the article is organised as follows. The theoretical framework is discussed in "The Theoretical Framework" section. In "Previous Studies" section we present previous studies. The hypotheses are presented in "The Hypotheses" section. In "The Data and Method" section we describe the data and the method. Descriptive statistics are provided in "Descriptive Statistics" section. The estimation results are presented in "The estimation results" section, while discussions and conclusions are given in "Discussions and Conclusions" section.

\section{The Theoretical Framework}

Within economic studies, the human capital theory is the traditional basis for analysing the relationship between education, skills and productivity. Expressed in its most general form, the theory deals with all relevant skills in the job context. If we have specific measures on skills and these have no effects on productivity, wages and the like, it can be argued that our measures are not valid expressions of human capital in the situation we are studying. Thus, the theory becomes empirically intangible. Then we 
think it is more interesting that the theory links productivity to formal education (Mincer 1958; Becker 1962). The theory rests on the assumption that individuals can invest in their human capital through formal education. Formal schooling has per se a positive effect on an individual's skills and thus productivity. The individual assesses the costs and benefits of different educational choices and selects an education that maximises the return on the labour market. In this manner, an educational choice becomes an individual investment in individual productivity.

Then we can imagine that this also applies to specific work tasks. An investment in individual productivity through education means that employees invest in their capacity to perform advanced work tasks. Thus, we can expect that there will be a positive correlation between formal education and the score on our measure of IPP. If we also have good indicators of cognitive skills, which we have, we can expect that skills partly mediate educational effects against potential productivity and partly that both education and skills directly affect IPP.

The signalling theory has a different perspective on educational choices and merits (Arrow 1973; Spence 1973). Based on this theory, formal education does not in itself have a positive effect on an individual's skills and productivity, but individuals signal skills and productivity through their education. Formal education serves instead as a selection arena for talent, skills and productivity. Both theories will predict that skills, productivity and formal education are positively correlated, but it is primarily the human capital theory that links these attributes causally. According to the signalling perspective on education and skills, one can expect that skills primarily will be positively related to productivity. Education will, at best, have indirect effects on productivity through its positive correlation with skills, or education may even appear as irrelevant when we have suitable measures on skills.

A more dynamic perspective on the relationship between age and productivity among employees that can be related to the human capital perspective, is represented by Lazear (1979) and Goldthorpe (2007). Lazear's (1979) starting point is that it is economically advantageous for both employers and employees to enter into long-term employment contracts. His theory states that it pays firms to agree to a long-term wage stream which pays workers less than the value of their marginal product (VMP) when young and more than their VMP when old. This will also be beneficial to the workers since their lifetime VMP will be higher than it would be in the absence of this payment schedule. On the other hand, Goldthorpe (2007) argues that this logic primarily covers relations to employees on 'service contracts' and not employment relations regulated by 'labour contracts'.

The wage level does not necessarily follow the productivity level over the life cycle, and perhaps this also applies to the allocation of advanced work tasks. Thus, employers give older employees access to work tasks that are also not strictly adapted to their productivity over the life cycle. In this area too, it may be important to distinguish between different types of employment contracts, e.g., that employees with a higher education in salaried jobs are to a greater extent allocated to work tasks with high productivity potential than modestly educated employees in manual jobs. According to such perspectives, we should expect that formal education and occupational status are central variables in explaining age variation in IPP in working life. The PIAAC data set has suitable indicators of occupational status and industrial affiliation. 
Finally, we should mention that there are other theories competing as explanations for such observations. Direct effects of formal education on employee IPP that are not captured by PIAAC's cognitive skills indicators can be understood as expressions of segmentation or educational credentialism in the labour market. In this case, formal education represents a gateway to specific activities or tasks in an employment relationship (Doeringer and Piore 1971; Collins 1979; Grøgaard and Børing 2017). Since we use occupation and industry as control variables, it is also possible that these variables act as carriers of possible gateway effects in the labour market.

Although we owe it to the reader to point out that there may be some diversity of interpretation, we choose here to relate hypotheses and empirical tests to central perspectives in human capital theory and signalling theory, and to the dynamic versions of these theories which can be linked to works by Lazear (1979) and Goldthorpe (2007).

\section{Previous Studies}

Several empirical studies have focused on how productivity is related to age. To the best of our knowledge, and in contrast to our analysis which is based on data for several countries, most of these studies are based on individual- or firm-level data for a single country. One exception is the paper by Aiyar et al. (2016), which is based on data covering up to 24 countries. They examine the relationship between workforce ageing and productivity in Europe. The data come from the OECD (and the Penn World Table 9.0). They find that an increase in the 55-64 age cohort of the labour force is associated with a reduction in total factor productivity.

Examples of studies that are based on matched employer-employee data for a single country are Hellerstein and Neumark (2004), Aubert and Crépon (2006), Lallemand and Rycx (2009), Van Ours (2009), Van Ours and Stoeldraijer (2011), Cataldi et al. (2012), Göbel and Zwick (2012), and Börsch-Supan and Weiss (2016). The results in several of these studies suggest that older workers are less productive than younger workers (Lallemand and Rycx 2009; Van Ours 2009; Van Ours and Stoeldraijer 2011; Cataldi et al. 2012), while this is hardly supported by some of the other studies (Aubert and Crépon 2006; Göbel and Zwick 2012; Börsch-Supan and Weiss 2016). Aubert and Crépon (2006) find that productivity increases with age until age 40 and then remains stable after this age, while the findings in Börsch-Supan and Weiss (2016) show that the average age-productivity profile of individual workers is increasing until age 65 . The results in Göbel and Zwick (2012) suggest that there are no pure age effects on productivity.

Skirbekk (2004) presents a literature survey on how individual productivity varies with age, and an overview over how employer-employee studies relate to age is presented in his article. For most of these studies, productivity is found to be concave in age. In another study, which is based on matched employer-employee data for the U.S., Hellerstein and Neumark (2004) conclude that both the estimated productivity profile and the estimated wage profile are concave in age. Estimating the relation between age and productivity potential, Skirbekk (2008) finds that productivity peaks for the 35-44 age group, but that the productivity peak shifts toward younger ages if the demand for experience falls. 
There are also several studies in the field of psychology on the relation of age to various dimensions of performance. Examples of such studies are Waldman and Avolio (1986), McEvoy and Cascio (1989), Sturman (2003), Kanfer and Ackerman (2004), and $\mathrm{Ng}$ and Feldman (2008). These studies show mixed results regarding the relationship between age and performance. Some of the studies give little support to the notion that work motivation declines with age (Kanfer and Ackerman 2004; Ng and Feldman 2008). Waldman and Avolio (1986) show a pattern of increases in performance, as measured by productivity indices, at higher ages. The analyses in McEvoy and Cascio (1989) reveal that age and job performance generally are unrelated. Sturman (2003) finds some support for the existence of an inverted U-shaped relationship between temporal variables (i.e. job experience, organisational tenure and employee age) and job performance.

Based on the presented employer-employee studies and the studies in the field of psychology above, it is not possible to draw any unambiguous conclusions about how productivity or the other different performance measures are related to age. The question is whether the mixed results can be explained on the basis of country-specific differences in some averages at the national level, where one of the averages may be the performance measure(s) used in the analysis. We are not aware of any studies that have examined this. As outlined in the introductory section, our contribution to the research literature is to first examine how age is related to our performance measure, i.e. the IPP score, and then to examine whether the estimated results can be explained by countryspecific differences in four averages at the national level. One of these averages is the average IPP score in each country.

\section{The Hypotheses}

Based on the studies in "Previous Studies" section, we are unable to draw unambiguous conclusions about the relationship between age and performance. However, in line with the studies using matched employer-employee data which find a negative relationship between productivity and age (Lallemand and Rycx 2009; Van Ours 2009; Van Ours and Stoeldraijer 2011; Cataldi et al. 2012), we thus formulate the following hypothesis:

H1: Older employees have a lower IPP than younger employees.

Based on the discussion of the signalling theory and the human capital theory in "The Theoretical Framework" section, we formulate the following two hypotheses regarding how IPP is related to educational level and skills level:

H2: IPP is positively related to (a) the educational level and (b) the skills level. H3: Variation in IPP is to a greater extent explained by the skills level than by the educational level.

If confidence in hypotheses $\mathrm{H} 2 \mathrm{~b}$ and $\mathrm{H} 3$ is strengthened, confidence in explanations based on signalling theory is also strengthened. If we find that both $\mathrm{H} 2 \mathrm{a}$ and $\mathrm{H} 2 \mathrm{~b}$ are supported, H3 will also be consistent with the human capital perspective on such 
relations. If we find support for $\mathrm{H} 2 \mathrm{a}$ and not for $\mathrm{H} 2 \mathrm{~b}$, we have strengthened the credibility of human capital theory at the expense of signalling theory.

In "The Estimation Results" section, we present two models, M1-M2. M1 measures the relationship between age and the IPP score. M2 controls this statistical relationship for formal education, documented skills and several other variables. Previous analyses of the PIAAC data have documented a systematic reduction in skills by age, which is interpreted as expressing a skill loss by age in most countries (Desjardins and Warnke 2012). Given that skills are expected to be positively related to IPP (H2b), we also expect that the inclusion of the skills level (together with the other control variables) in M2 reduces the correlation between the IPP score and age compared to M1. We therefore formulate the following hypothesis:

H4: (a) The inclusion of the control variables in M2 reduces the relationship between the IPP score and age compared to M1, and (b) the employee's profession is one of the most important control variables.

If the inclusion of control variables in M2 reduces the statistical effect of age on IPP, control variables with significant effects on IPP function as mediators of age differences in M1 (the bivariate case). The reason for this is that age is an underlying variable, and the control variables are intermediate variables, in $\mathrm{M} 2$. If $\mathrm{H} 4 \mathrm{~b}$ is strengthened without support for $\mathrm{H} 4 \mathrm{a}$, we can argue that the allocation of learning and demanding work tasks is linked to the employee's profession, i.e. this allocation depends on whether a person's employment is regulated by a service or labour contract (cf. Goldthorpe 2007). Here, occupation serves as an explanatory variable in addition to age in M2. If the profession mediates age differences in the sense that both $\mathrm{H} 4 \mathrm{a}$ and $\mathrm{H} 4 \mathrm{~b}$ are supported, Goldthorpe's clarification of Lazear's theory is strengthened (Lazear 1979; Goldthorpe 2007). In this case it is only rational for employers to enter into long-term employment contracts with employees in specific positions, the so-called 'service contracts'. For older workers, we imagine that if this is the case with wages, it also applies to the allocation of learning and demanding work tasks as measured by our IPP index.

The hypotheses H1-H4 will be tested in the analysis.

\section{The Data and Method}

In the analysis, we use individual-level data from the PIAAC database. The data is collected using personal interviews. Basically, 200,588 persons in 31 countries are included in the data set. Four of the 31 countries (Austria, Canada, Germany, the US) are excluded from the sample since all values are missing for at least one of the explanatory variables. This reduces the sample to 158,300 persons. All the remaining 27 countries are included in the sample.

We only include employees and not self-employed in the sample. The reasons are that dummy variables for firm size are included as control variables in the regressions, 
and firm size only refers to employees. Persons with missing values for at least one of the explanatory variables are excluded from the sample, with two exceptions: persons with unknown educational level or firm size are included in the sample. The sample is also limited to the age group 20-59 years. However, most countries have a formal retirement age that is higher than 59 years.

Table 1 Descriptive statistics: four national averages, by country

\begin{tabular}{|c|c|c|c|c|c|}
\hline Country & $\begin{array}{l}\text { Average } \\
\text { IPP score }\end{array}$ & $\begin{array}{l}\text { Average skill loss for } \\
\text { the oldest age group }\end{array}$ & $\begin{array}{l}\text { Average skills level } \\
\text { for all age groups }\end{array}$ & $\begin{array}{l}\text { Proportion of employees } \\
\text { with higher education }\end{array}$ & $\mathrm{N}$ \\
\hline Belgium & 15.4 & 53.6 & 282.0 & 43.4 & 2754 \\
\hline Chile & 16.8 & 64.7 & 227.5 & 33.1 & 2223 \\
\hline Cyprus & 15.0 & 2.6 & 274.3 & 45.1 & 2230 \\
\hline Czech Republic & 14.4 & 45.9 & 277.7 & 22.0 & 2688 \\
\hline Denmark & 16.8 & 41.3 & 278.9 & 43.3 & 3941 \\
\hline Estonia & 16.3 & 50.4 & 279.9 & 44.3 & 4172 \\
\hline Finland & 17.6 & 63.4 & 299.5 & 48.2 & 2994 \\
\hline France & 15.6 & 69.1 & 268.2 & 34.9 & 3584 \\
\hline Greece & 14.4 & 13.0 & 258.0 & 36.5 & 1433 \\
\hline Ireland & 16.4 & 42.6 & 275.3 & 46.4 & 2767 \\
\hline Israel & 15.8 & 40.5 & 264.5 & 52.9 & 2576 \\
\hline Italy & 14.9 & 12.5 & 254.3 & 16.5 & 2051 \\
\hline Japan & 15.3 & 42.2 & 303.2 & 50.0 & 3001 \\
\hline $\begin{array}{l}\text { Republic of } \\
\text { Korea }\end{array}$ & 13.7 & 91.1 & 276.1 & 46.7 & 2975 \\
\hline Lithuania & 13.6 & 58.3 & 270.6 & 35.4 & 2606 \\
\hline Netherlands & 16.0 & 63.2 & 292.2 & 36.1 & 2939 \\
\hline New Zealand & 18.0 & 15.0 & 286.6 & 50.2 & 3050 \\
\hline Norway & 17.6 & 28.1 & 286.7 & 44.1 & 2958 \\
\hline Poland & 14.8 & 41.1 & 273.7 & 38.3 & 3965 \\
\hline $\begin{array}{l}\text { Russian } \\
\text { Federation }\end{array}$ & 14.1 & -5.8 & 279.9 & 69.7 & 1680 \\
\hline Singapore & 16.0 & 107.9 & 262.3 & 58.2 & 3055 \\
\hline $\begin{array}{l}\text { Slovak } \\
\text { Republic }\end{array}$ & 14.6 & 36.7 & 280.4 & 26.1 & 2572 \\
\hline Slovenia & 16.6 & 37.1 & 261.4 & 33.2 & 2544 \\
\hline Spain & 16.4 & 38.7 & 261.9 & 41.6 & 2543 \\
\hline Sweden & 17.4 & 43.2 & 290.2 & 35.5 & 2570 \\
\hline Turkey & 13.7 & 37.7 & 237.3 & 26.9 & 1543 \\
\hline $\begin{array}{l}\text { United } \\
\text { Kingdom }\end{array}$ & 16.9 & 26.8 & 280.8 & 44.2 & 4363 \\
\hline
\end{tabular}


Table 2 Estimated effects on the IPP score, by country

\begin{tabular}{|c|c|c|c|c|c|c|c|c|c|c|c|c|}
\hline \multirow{2}{*}{$\begin{array}{l}\text { Explanatory } \\
\text { variables }\end{array}$} & \multicolumn{2}{|l|}{ M1 } & \multicolumn{2}{|l|}{ M2 } & \multicolumn{2}{|l|}{ M1 } & \multicolumn{2}{|l|}{ M2 } & \multicolumn{2}{|l|}{ M1 } & \multicolumn{2}{|l|}{ M2 } \\
\hline & $\mathrm{C}$ & SE & $\mathrm{C}$ & SE & $\mathrm{C}$ & SE & $\mathrm{C}$ & SE & $\mathrm{C}$ & SE & $\mathrm{C}$ & $\mathrm{SE}$ \\
\hline & \multicolumn{4}{|c|}{ Belgium } & \multicolumn{4}{|c|}{ Chile } & \multicolumn{4}{|c|}{ Cyprus } \\
\hline Aged 20-29 & 0.22 & 0.27 & 0.28 & 0.29 & 0.57 & 0.44 & 0.88 & 0.46 & 0.09 & 0.37 & 0.28 & 0.38 \\
\hline Aged 40-49 & -0.68 & $* 0.29$ & -0.27 & 0.29 & -0.25 & 0.45 & 0.08 & 0.39 & -0.31 & 0.31 & 0.02 & 0.33 \\
\hline Aged 50-59 & -1.51 & $* 0.25$ & -0.41 & 0.38 & -0.84 & 0.51 & 0.05 & 0.51 & -1.43 & $* 0.34$ & -0.48 & 0.53 \\
\hline $\begin{array}{l}\text { Low. secon. } \\
\text { Less }\end{array}$ & & & -0.76 & $* 0.29$ & & & -0.55 & 0.46 & & & -0.06 & 0.35 \\
\hline $\begin{array}{l}\text { Higher } \\
\text { education }\end{array}$ & & & 0.44 & $* 0.20$ & & & 0.41 & 0.27 & & & 0.77 & $* 0.23$ \\
\hline $\begin{array}{l}\text { Unkn. educ. } \\
\text { Lev. }\end{array}$ & & & -1.18 & 1.20 & & & & & & & & \\
\hline Skills level & & & 0.00 & 0.00 & & & -0.01 & $* 0.00$ & & & -0.01 & $* 0.00$ \\
\hline \multicolumn{13}{|l|}{ Occupation } \\
\hline Armed forces & & & 1.58 & 1.30 & & & 3.39 & $* 1.23$ & & & 1.81 & $* 0.73$ \\
\hline Managers & & & 0.49 & 0.28 & & & 0.81 & 0.75 & & & 1.28 & $* 0.54$ \\
\hline $\begin{array}{l}\text { Techn., ass. } \\
\text { Prof. }\end{array}$ & & & -0.51 & 0.26 & & & -0.13 & 0.34 & & & -0.28 & 0.33 \\
\hline Clerical support & & & -1.76 & $* 0.31$ & & & -1.31 & $* 0.56$ & & & -1.39 & $* 0.42$ \\
\hline $\begin{array}{l}\text { Service and } \\
\text { sales }\end{array}$ & & & -0.77 & $* 0.31$ & & & -0.33 & 0.51 & & & -0.62 & 0.38 \\
\hline $\begin{array}{l}\text { Agricul., } \\
\text { forestry }\end{array}$ & & & 0.29 & 0.83 & & & -4.75 & $* 1.04$ & & & 0.51 & 1.60 \\
\hline $\begin{array}{l}\text { Craft, rela. } \\
\text { Trades }\end{array}$ & & & -2.00 & $* 0.36$ & & & -1.09 & 0.76 & & & -1.29 & $* 0.48$ \\
\hline Plant, machine & & & -3.78 & $* 0.43$ & & & -4.02 & $* 0.59$ & & & -3.20 & $* 0.65$ \\
\hline Elementary & & & -4.44 & $* 0.37$ & & & -3.95 & $* 0.64$ & & & -3.70 & $* 0.59$ \\
\hline Unknown & & & -2.35 & $* 0.66$ & & & & & & & -0.52 & 0.80 \\
\hline \multirow[t]{2}{*}{$\mathrm{N}$} & \multicolumn{4}{|c|}{2754} & \multicolumn{4}{|c|}{2223} & \multicolumn{4}{|c|}{2230} \\
\hline & \multicolumn{4}{|c|}{ Czech Republic } & \multicolumn{4}{|c|}{ Denmark } & \multicolumn{4}{|c|}{ Estonia } \\
\hline Aged 20-29 & 0.02 & 0.26 & 0.11 & 0.29 & -1.00 & $* 0.24$ & 0.47 & 0.24 & -0.30 & 0.17 & 0.13 & 0.21 \\
\hline Aged $40-49$ & -0.96 & $* 0.31$ & -0.22 & 0.38 & -0.18 & 0.20 & -0.59 & $* 0.23$ & -0.88 & $* 0.16$ & -0.50 & $* 0.20$ \\
\hline Aged 50-59 & -1.03 & *0.34 & 0.18 & 0.54 & -0.86 & $* 0.22$ & -1.33 & $* 0.35$ & -1.49 & $* 0.19$ & -0.92 & $* 0.29$ \\
\hline $\begin{array}{l}\text { Low. secon. } \\
\text { Less }\end{array}$ & & & -0.89 & 0.47 & & & -0.92 & $* 0.20$ & & & -0.56 & $* 0.24$ \\
\hline $\begin{array}{l}\text { Higher } \\
\text { education }\end{array}$ & & & 0.75 & $* 0.33$ & & & 0.57 & $* 0.19$ & & & 0.37 & $* 0.17$ \\
\hline $\begin{array}{l}\text { Unkn. educ. } \\
\text { Lev. }\end{array}$ & & & 1.45 & 7.28 & & & & & & & & \\
\hline Skills level & & & 0.01 & 0.00 & & & -0.00 & 0.00 & & & -0.01 & $* 0.00$ \\
\hline \multicolumn{13}{|l|}{ Occupation } \\
\hline Armed forces & & & -0.09 & 1.61 & & & -1.55 & 0.98 & & & 0.28 & 0.98 \\
\hline \multirow[t]{2}{*}{ Managers } & & & 1.76 & $* 0.43$ & & & -0.24 & 0.23 & & & 1.51 & $* 0.24$ \\
\hline & & & 0.37 & 0.38 & & & -0.67 & $* 0.21$ & & & 0.05 & 0.22 \\
\hline
\end{tabular}


Table 2 (continued)

\begin{tabular}{|c|c|c|c|c|c|c|c|c|c|c|c|c|}
\hline \multirow{2}{*}{$\begin{array}{l}\text { Explanatory } \\
\text { variables }\end{array}$} & \multicolumn{2}{|l|}{ M1 } & \multicolumn{2}{|l|}{ M2 } & \multicolumn{2}{|l|}{ M1 } & \multicolumn{2}{|l|}{ M2 } & \multicolumn{2}{|l|}{ M1 } & \multicolumn{2}{|l|}{ M2 } \\
\hline & $\mathrm{C}$ & SE & $\mathrm{C}$ & SE & $\mathrm{C}$ & SE & $\mathrm{C}$ & SE & $\mathrm{C}$ & SE & $\mathrm{C}$ & SE \\
\hline \multicolumn{13}{|l|}{$\begin{array}{c}\text { Techn., ass. } \\
\text { Prof. }\end{array}$} \\
\hline Clerical support & & & -0.26 & 0.45 & & & -2.35 & $* 0.27$ & & & -1.31 & $* 0.32$ \\
\hline $\begin{array}{l}\text { Service and } \\
\text { sales }\end{array}$ & & & 0.35 & 0.42 & & & -0.64 & $* 0.26$ & & & -0.65 & $* 0.26$ \\
\hline $\begin{array}{l}\text { Agricul., } \\
\text { forestry }\end{array}$ & & & -2.58 & 1.48 & & & -2.50 & $* 0.74$ & & & -1.07 & 0.73 \\
\hline $\begin{array}{l}\text { Craft, rela. } \\
\text { Trades }\end{array}$ & & & -0.48 & 0.54 & & & -2.08 & $* 0.32$ & & & -1.63 & $* 0.32$ \\
\hline Plant, machine & & & -2.36 & $* 0.44$ & & & -3.47 & $* 0.42$ & & & -3.59 & $* 0.29$ \\
\hline Elementary & & & -2.42 & $* 0.67$ & & & -4.17 & $* 0.36$ & & & -4.58 & $* 0.31$ \\
\hline Unknown & & & -1.08 & 3.14 & & & & & & & 2.36 & $* 0.69$ \\
\hline \multirow[t]{2}{*}{$\mathrm{N}$} & \multicolumn{4}{|c|}{2688} & \multicolumn{4}{|c|}{3941} & \multicolumn{4}{|c|}{4172} \\
\hline & \multicolumn{4}{|c|}{ Finland } & \multicolumn{4}{|c|}{ France } & \multicolumn{4}{|c|}{ Greece } \\
\hline Aged 20-29 & -0.46 & $* 0.20$ & 0.61 & $* 0.20$ & 0.45 & $* 0.18$ & 0.87 & $* 0.20$ & 0.27 & 0.40 & 0.25 & 0.44 \\
\hline Aged $40-49$ & -0.56 & $* 0.20$ & -1.00 & $* 0.22$ & -0.87 & $* 0.18$ & -0.51 & $* 0.23$ & -0.83 & $* 0.40$ & -0.33 & 0.40 \\
\hline Aged $50-59$ & -0.90 & $* 0.19$ & -1.54 & $* 0.31$ & -1.54 & $* 0.21$ & -1.08 & $* 0.33$ & -1.49 & $* 0.52$ & -0.32 & 0.55 \\
\hline $\begin{array}{l}\text { Low. secon. } \\
\text { Less }\end{array}$ & & & -0.98 & $* 0.31$ & & & -0.95 & $* 0.21$ & & & -0.73 & 0.43 \\
\hline $\begin{array}{l}\text { Higher } \\
\text { education }\end{array}$ & & & 0.09 & 0.18 & & & -0.13 & 0.19 & & & -0.24 & 0.37 \\
\hline $\begin{array}{l}\text { Unkn. educ. } \\
\text { Lev. }\end{array}$ & & & & & & & -0.05 & 0.30 & & & & \\
\hline Skills level & & & -0.00 & $* 0.00$ & & & 0.00 & 0.00 & & & -0.00 & 0.00 \\
\hline \multicolumn{13}{|l|}{ Occupation } \\
\hline Armed forces & & & 1.91 & $* 0.85$ & & & 0.75 & 0.64 & & & -2.52 & $* 0.95$ \\
\hline Managers & & & 0.54 & 0.28 & & & 0.12 & 0.22 & & & -0.44 & 0.97 \\
\hline $\begin{array}{l}\text { Techn., ass. } \\
\text { Prof. }\end{array}$ & & & 0.33 & 0.22 & & & -0.64 & $* 0.21$ & & & -2.17 & $* 0.61$ \\
\hline Clerical support & & & -0.73 & $* 0.27$ & & & -2.17 & $* 0.27$ & & & -1.85 & $* 0.73$ \\
\hline $\begin{array}{l}\text { Service and } \\
\text { sales }\end{array}$ & & & -0.21 & 0.28 & & & -1.61 & $* 0.25$ & & & -2.43 & $* 0.71$ \\
\hline $\begin{array}{l}\text { Agricul., } \\
\text { forestry }\end{array}$ & & & -3.94 & $* 0.98$ & & & -4.27 & $* 0.83$ & & & -2.95 & $* 1.44$ \\
\hline $\begin{array}{c}\text { Craft, rela. } \\
\text { Trades }\end{array}$ & & & -2.11 & $* 0.34$ & & & -3.23 & $* 0.32$ & & & -3.46 & $* 0.98$ \\
\hline Plant, machine & & & -2.95 & $* 0.33$ & & & -4.54 & $* 0.28$ & & & -4.85 & $* 0.88$ \\
\hline Elementary & & & -2.74 & $* 0.38$ & & & -4.64 & $* 0.27$ & & & -5.58 & $* 0.76$ \\
\hline Unknown & & & & & & & & & & & -2.21 & 1.28 \\
\hline \multirow[t]{2}{*}{$\mathrm{N}$} & \multicolumn{4}{|c|}{2994} & \multicolumn{4}{|c|}{3584} & \multicolumn{4}{|c|}{1433} \\
\hline & \multicolumn{4}{|c|}{ Ireland } & \multicolumn{4}{|c|}{ Israel } & \multicolumn{4}{|c|}{ Italy } \\
\hline Aged 20-29 & -0.58 & 0.30 & 0.29 & 0.31 & -0.66 & $* 0.22$ & 0.28 & 0.28 & -0.18 & 0.38 & -0.08 & 0.39 \\
\hline Aged 40-49 & -0.76 & $* 0.25$ & -0.74 & $* 0.29$ & -0.57 & $* 0.28$ & -0.31 & 0.28 & -0.63 & 0.33 & -0.92 & $* 0.31$ \\
\hline
\end{tabular}


Table 2 (continued)

\begin{tabular}{|c|c|c|c|c|c|c|c|c|c|c|c|c|}
\hline \multirow{2}{*}{$\begin{array}{l}\text { Explanatory } \\
\text { variables }\end{array}$} & \multicolumn{2}{|l|}{ M1 } & \multicolumn{2}{|l|}{ M2 } & \multicolumn{2}{|l|}{ M1 } & \multicolumn{2}{|l|}{ M2 } & \multicolumn{2}{|l|}{ M1 } & \multicolumn{2}{|l|}{ M2 } \\
\hline & $\mathrm{C}$ & $\mathrm{SE}$ & $\mathrm{C}$ & SE & $\mathrm{C}$ & $\mathrm{SE}$ & $\mathrm{C}$ & $\mathrm{SE}$ & $\mathrm{C}$ & $\mathrm{SE}$ & $\mathrm{C}$ & $\mathrm{SE}$ \\
\hline Aged $50-59$ & -2.33 & $* 0.35$ & -2.22 & $* 0.46$ & -1.36 & $* 0.32$ & -0.52 & 0.43 & -1.20 & $* 0.35$ & -1.93 & $* 0.39$ \\
\hline $\begin{array}{l}\text { Low. secon. } \\
\text { Less }\end{array}$ & & & -0.26 & 0.33 & & & -1.36 & $* 0.42$ & & & -0.75 & $* 0.32$ \\
\hline $\begin{array}{l}\text { Higher } \\
\text { education }\end{array}$ & & & 0.72 & $* 0.26$ & & & 0.46 & 0.26 & & & 0.65 & $* 0.33$ \\
\hline $\begin{array}{l}\text { Unkn. educ. } \\
\text { Lev. }\end{array}$ & & & -3.16 & $* 0.72$ & & & & & & & & \\
\hline Skills level & & & -0.00 & 0.00 & & & -0.00 & 0.00 & & & -0.00 & 0.00 \\
\hline \multicolumn{13}{|l|}{ Occupation } \\
\hline Armed forces & & & -2.01 & 1.70 & & & & & & & 1.98 & 1.02 \\
\hline Managers & & & -0.19 & 0.42 & & & 0.47 & 0.34 & & & 2.08 & $* 0.66$ \\
\hline $\begin{array}{l}\text { Techn., ass. } \\
\text { Prof. }\end{array}$ & & & -0.70 & $* 0.31$ & & & -0.76 & $* 0.35$ & & & -0.99 & $* 0.30$ \\
\hline Clerical support & & & -1.89 & $* 0.30$ & & & -1.52 & $* 0.43$ & & & -2.19 & $* 0.43$ \\
\hline $\begin{array}{l}\text { Service and } \\
\text { sales }\end{array}$ & & & -1.80 & $* 0.41$ & & & -2.09 & $* 0.35$ & & & -1.55 & $* 0.49$ \\
\hline $\begin{array}{l}\text { Agricul., } \\
\text { forestry }\end{array}$ & & & -2.72 & $* 0.78$ & & & -3.27 & $* 0.94$ & & & -4.39 & $* 1.11$ \\
\hline $\begin{array}{l}\text { Craft, rela. } \\
\text { Trades }\end{array}$ & & & -2.14 & $* 0.45$ & & & -2.06 & $* 0.53$ & & & -2.15 & $* 0.49$ \\
\hline Plant, machine & & & -4.02 & $* 0.60$ & & & -3.05 & $* 0.58$ & & & -3.53 & $* 0.54$ \\
\hline Elementary & & & -4.54 & $* 0.52$ & & & -5.64 & $* 0.61$ & & & -4.62 & $* 0.46$ \\
\hline Unknown & & & -5.02 & 3.93 & & & -1.10 & 0.77 & & & & \\
\hline \multirow[t]{2}{*}{$\mathrm{N}$} & \multicolumn{4}{|c|}{2767} & \multicolumn{4}{|c|}{2576} & \multicolumn{4}{|c|}{2051} \\
\hline & \multicolumn{4}{|c|}{ Japan } & \multicolumn{4}{|c|}{ Republic of Korea } & \multicolumn{4}{|c|}{ Lithuania } \\
\hline Aged 20-29 & 0.19 & 0.20 & 0.18 & 0.22 & 0.14 & 0.25 & 0.44 & 0.26 & 0.75 & $* 0.34$ & 0.64 & $* 0.27$ \\
\hline Aged 40-49 & -0.68 & $* 0.22$ & -0.97 & $* 0.22$ & -1.38 & $* 0.22$ & -0.82 & $* 0.23$ & -1.26 & $* 0.34$ & -1.08 & $* 0.33$ \\
\hline Aged 50-59 & -0.96 & $* 0.21$ & -1.29 & $* 0.26$ & -2.67 & $* 0.24$ & -1.67 & $* 0.30$ & -1.90 & $* 0.29$ & -1.39 & $* 0.45$ \\
\hline $\begin{array}{l}\text { Low. secon. } \\
\text { Less }\end{array}$ & & & -0.03 & 0.30 & & & -1.28 & $* 0.33$ & & & -0.13 & 0.54 \\
\hline $\begin{array}{l}\text { Higher } \\
\text { education }\end{array}$ & & & 0.02 & 0.20 & & & 0.18 & 0.23 & & & 0.58 & $* 0.28$ \\
\hline $\begin{array}{l}\text { Unkn. educ. } \\
\text { Lev. }\end{array}$ & & & 3.60 & $* 0.41$ & & & & & & & 0.56 & 0.53 \\
\hline Skills level & & & 0.00 & 0.00 & & & -0.00 & 0.00 & & & -0.00 & 0.00 \\
\hline \multicolumn{13}{|l|}{ Occupation } \\
\hline Armed forces & & & -0.77 & 1.15 & & & 0.97 & 0.73 & & & 3.28 & $* 1.14$ \\
\hline Managers & & & 0.83 & $* 0.32$ & & & 0.21 & 0.49 & & & 0.78 & 0.45 \\
\hline $\begin{array}{l}\text { Techn., ass. } \\
\text { Prof. }\end{array}$ & & & -0.61 & $* 0.25$ & & & -0.68 & $* 0.32$ & & & -0.02 & 0.38 \\
\hline Clerical support & & & -2.27 & $* 0.26$ & & & -1.54 & $* 0.28$ & & & -1.02 & $* 0.41$ \\
\hline $\begin{array}{l}\text { Service and } \\
\text { sales }\end{array}$ & & & -0.36 & 0.27 & & & -1.34 & $* 0.30$ & & & -1.55 & $* 0.38$ \\
\hline
\end{tabular}


Table 2 (continued)

\begin{tabular}{|c|c|c|c|c|c|c|c|c|c|c|c|c|}
\hline \multirow{2}{*}{$\begin{array}{l}\text { Explanatory } \\
\text { variables }\end{array}$} & \multicolumn{2}{|l|}{ M1 } & \multicolumn{2}{|l|}{ M2 } & \multicolumn{2}{|l|}{ M1 } & \multicolumn{2}{|l|}{ M2 } & \multicolumn{2}{|l|}{ M1 } & \multicolumn{2}{|l|}{ M2 } \\
\hline & $\mathrm{C}$ & $\mathrm{SE}$ & $\mathrm{C}$ & $\mathrm{SE}$ & $\mathrm{C}$ & $\mathrm{SE}$ & $\mathrm{C}$ & SE & $\mathrm{C}$ & SE & $\mathrm{C}$ & SE \\
\hline $\begin{array}{l}\text { Agricul., } \\
\text { forestry }\end{array}$ & & & -2.24 & $* 1.11$ & & & -0.52 & 1.21 & & & -3.01 & $* 1.16$ \\
\hline $\begin{array}{l}\text { Craft, rela. } \\
\text { Trades }\end{array}$ & & & -1.20 & $* 0.35$ & & & -1.63 & $* 0.39$ & & & -2.50 & $* 0.43$ \\
\hline Plant, machine & & & -2.53 & $* 0.37$ & & & -2.92 & $* 0.37$ & & & -4.31 & $* 0.44$ \\
\hline Elementary & & & -2.58 & $* 0.45$ & & & -3.14 & $* 0.40$ & & & -4.52 & $* 0.46$ \\
\hline Unknown & & & -0.83 & 1.56 & & & -1.77 & 6.25 & & & 3.28 & $* 1.63$ \\
\hline \multirow[t]{2}{*}{$\mathrm{N}$} & \multicolumn{4}{|c|}{3001} & \multicolumn{4}{|c|}{2975} & \multicolumn{4}{|c|}{2606} \\
\hline & \multicolumn{4}{|c|}{ Netherlands } & \multicolumn{4}{|c|}{ New Zealand } & \multicolumn{4}{|c|}{ Norway } \\
\hline Aged 20-29 & -0.12 & 0.23 & 0.82 & $* 0.23$ & 0.18 & 0.26 & 0.40 & 0.27 & -0.70 & $* 0.18$ & 0.11 & 0.18 \\
\hline Aged 40-49 & -0.55 & $* 0.26$ & -0.60 & $* 0.24$ & -0.58 & $* 0.23$ & -0.36 & 0.25 & -0.01 & 0.19 & -0.21 & 0.21 \\
\hline Aged 50-59 & -1.04 & $* 0.26$ & -1.34 & $* 0.33$ & -0.61 & $* 0.30$ & -0.24 & 0.35 & -0.58 & $* 0.19$ & -0.85 & $* 0.29$ \\
\hline $\begin{array}{l}\text { Low. secon. } \\
\text { Less }\end{array}$ & & & -0.74 & $* 0.22$ & & & -0.43 & 0.27 & & & -0.42 & $* 0.21$ \\
\hline $\begin{array}{l}\text { Higher } \\
\text { education }\end{array}$ & & & 0.13 & 0.19 & & & -0.08 & 0.22 & & & 0.27 & 0.17 \\
\hline $\begin{array}{l}\text { Unkn. educ. } \\
\text { Lev. }\end{array}$ & & & & & & & -0.28 & 1.51 & & & 0.23 & 0.43 \\
\hline Skills level & & & 0.00 & 0.00 & & & -0.01 & $* 0.00$ & & & -0.00 & 0.00 \\
\hline \multicolumn{13}{|l|}{ Occupation } \\
\hline Armed forces & & & 1.40 & 1.16 & & & 0.78 & 0.65 & & & & \\
\hline Managers & & & 0.39 & 0.22 & & & -0.27 & 0.25 & & & 0.05 & 0.23 \\
\hline $\begin{array}{l}\text { Techn., ass. } \\
\text { Prof. }\end{array}$ & & & -0.70 & $* 0.22$ & & & -1.03 & $* 0.24$ & & & -0.77 & $* 0.20$ \\
\hline Clerical support & & & -2.49 & $* 0.31$ & & & -2.07 & $* 0.32$ & & & -2.30 & $* 0.31$ \\
\hline $\begin{array}{l}\text { Service and } \\
\text { sales }\end{array}$ & & & -1.20 & $* 0.34$ & & & -1.78 & $* 0.34$ & & & -0.52 & $* 0.23$ \\
\hline $\begin{array}{l}\text { Agricul., } \\
\text { forestry }\end{array}$ & & & -3.65 & $* 0.76$ & & & -3.64 & $* 0.65$ & & & -3.61 & $* 1.71$ \\
\hline $\begin{array}{l}\text { Craft, rela. } \\
\text { Trades }\end{array}$ & & & -2.74 & $* 0.37$ & & & -1.85 & $* 0.33$ & & & -1.20 & $* 0.32$ \\
\hline Plant, machine & & & -4.28 & $* 0.44$ & & & -3.77 & $* 0.50$ & & & -3.19 & $* 0.48$ \\
\hline Elementary & & & -4.86 & $* 0.39$ & & & -5.27 & $* 0.52$ & & & -4.02 & $* 0.40$ \\
\hline Unknown & & & & & & & -6.94 & $* 1.18$ & & & -1.12 & $* 0.26$ \\
\hline \multirow[t]{2}{*}{$\mathrm{N}$} & \multicolumn{4}{|c|}{2939} & \multicolumn{4}{|c|}{3050} & \multicolumn{4}{|c|}{2958} \\
\hline & \multicolumn{4}{|c|}{ Poland } & \multicolumn{4}{|c|}{ Russian Federation } & \multicolumn{4}{|c|}{ Singapore } \\
\hline Aged 20-29 & 0.39 & 0.29 & 0.51 & 0.30 & 0.63 & 0.52 & 0.57 & 0.57 & 0.24 & 0.23 & 0.31 & 0.21 \\
\hline Aged 40-49 & -0.63 & 0.40 & -0.14 & 0.38 & -0.84 & 0.65 & -0.69 & 0.57 & -1.38 & $* 0.20$ & -0.40 & 0.24 \\
\hline Aged 50-59 & -1.99 & $* 0.35$ & -0.94 & $* 0.43$ & -2.25 & $* 0.54$ & -1.57 & 0.91 & -3.26 & $* 0.23$ & -1.23 & $* 0.38$ \\
\hline $\begin{array}{l}\text { Low. secon. } \\
\text { Less }\end{array}$ & & & -0.67 & 0.47 & & & -1.12 & 1.15 & & & -1.22 & $* 0.38$ \\
\hline $\begin{array}{l}\text { Higher } \\
\text { education }\end{array}$ & & & 0.26 & 0.25 & & & 0.99 & $* 0.36$ & & & 0.14 & 0.27 \\
\hline
\end{tabular}


Table 2 (continued)

\begin{tabular}{|c|c|c|c|c|c|c|c|c|c|c|c|c|}
\hline \multirow{2}{*}{$\begin{array}{l}\text { Explanatory } \\
\text { variables }\end{array}$} & \multicolumn{2}{|l|}{ M1 } & \multicolumn{2}{|l|}{ M2 } & \multicolumn{2}{|l|}{ M1 } & \multicolumn{2}{|l|}{ M2 } & \multicolumn{2}{|l|}{ M1 } & \multicolumn{2}{|l|}{ M2 } \\
\hline & $\mathrm{C}$ & SE & $\mathrm{C}$ & SE & $\mathrm{C}$ & SE & $\mathrm{C}$ & SE & $\mathrm{C}$ & SE & $\mathrm{C}$ & SE \\
\hline \multicolumn{13}{|l|}{$\begin{array}{l}\text { Unkn. educ. } \\
\text { Lev. }\end{array}$} \\
\hline Skills level & & & -0.00 & 0.00 & & & 0.00 & 0.00 & & & 0.00 & 0.00 \\
\hline \multicolumn{13}{|l|}{ Occupation } \\
\hline Armed forces & & & -0.40 & 0.99 & & & 1.96 & 1.88 & & & 0.56 & 0.62 \\
\hline Managers & & & 0.23 & 0.42 & & & 1.46 & $* 0.61$ & & & 1.06 & $* 0.28$ \\
\hline $\begin{array}{l}\text { Techn., ass. } \\
\text { Prof. }\end{array}$ & & & -0.90 & $* 0.35$ & & & -0.81 & 0.67 & & & -0.20 & 0.25 \\
\hline Clerical support & & & -2.59 & $* 0.43$ & & & -0.94 & 0.53 & & & -1.46 & $* 0.40$ \\
\hline $\begin{array}{l}\text { Service and } \\
\text { sales }\end{array}$ & & & -1.43 & $* 0.34$ & & & -1.99 & $* 0.35$ & & & -0.08 & 0.45 \\
\hline $\begin{array}{l}\text { Agricul., } \\
\text { forestry }\end{array}$ & & & -4.09 & $* 1.53$ & & & -3.13 & 2.60 & & & & \\
\hline $\begin{array}{l}\text { Craft, rela. } \\
\text { Trades }\end{array}$ & & & -3.24 & $* 0.39$ & & & -3.48 & $* 0.76$ & & & -0.88 & 0.57 \\
\hline Plant, machine & & & -4.96 & $* 0.43$ & & & -5.35 & $* 0.58$ & & & -3.14 & $* 0.59$ \\
\hline Elementary & & & -5.93 & $* 0.50$ & & & -6.51 & $* 0.53$ & & & -3.34 & $* 0.63$ \\
\hline Unknown & & & 3.51 & 1.86 & & & & & & & & \\
\hline \multirow[t]{2}{*}{$\mathrm{N}$} & \multicolumn{4}{|c|}{3965} & \multicolumn{4}{|c|}{1680} & \multicolumn{4}{|c|}{3055} \\
\hline & \multicolumn{4}{|c|}{ Slovakia } & \multicolumn{4}{|c|}{ Slovenia } & \multicolumn{4}{|c|}{ Spain } \\
\hline Aged 20-29 & 0.30 & 0.32 & 0.56 & 0.31 & 0.01 & 0.31 & 0.85 & $* 0.30$ & -0.07 & 0.29 & 0.70 & $* 0.29$ \\
\hline Aged 40-49 & -0.58 & $* 0.28$ & -0.61 & $* 0.29$ & -0.87 & $* 0.25$ & -1.28 & $* 0.31$ & -0.38 & 0.24 & -0.56 & $* 0.24$ \\
\hline Aged $50-59$ & -1.30 & $* 0.28$ & -1.21 & $* 0.41$ & -1.32 & $* 0.24$ & -2.07 & $* 0.45$ & -0.54 & 0.36 & -1.30 & $* 0.39$ \\
\hline $\begin{array}{l}\text { Low. secon. } \\
\text { Less }\end{array}$ & & & -1.48 & $* 0.33$ & & & -1.35 & $* 0.31$ & & & -1.19 & $* 0.31$ \\
\hline $\begin{array}{l}\text { Higher } \\
\text { education }\end{array}$ & & & 0.51 & 0.28 & & & 0.43 & 0.24 & & & -0.13 & 0.28 \\
\hline \multicolumn{13}{|l|}{$\begin{array}{l}\text { Unkn. educ. } \\
\text { Lev. }\end{array}$} \\
\hline Skills level & & & 0.01 & 0.00 & & & -0.00 & 0.00 & & & 0.01 & $* 0.00$ \\
\hline \multicolumn{13}{|l|}{ Occupation } \\
\hline Armed forces & & & 0.41 & 1.20 & & & -1.23 & 1.08 & & & & \\
\hline Managers & & & 0.87 & 0.49 & & & 1.01 & $* 0.29$ & & & -0.12 & 0.56 \\
\hline $\begin{array}{l}\text { Techn., ass. } \\
\text { Prof. }\end{array}$ & & & -0.54 & 0.34 & & & -0.71 & $* 0.30$ & & & -1.19 & $* 0.37$ \\
\hline Clerical support & & & -2.13 & $* 0.40$ & & & -1.91 & $* 0.39$ & & & -2.81 & $* 0.38$ \\
\hline $\begin{array}{l}\text { Service and } \\
\text { sales }\end{array}$ & & & -1.64 & $* 0.39$ & & & -1.01 & $* 0.36$ & & & -2.25 & $* 0.42$ \\
\hline $\begin{array}{l}\text { Agricul., } \\
\text { forestry }\end{array}$ & & & -3.92 & $* 1.15$ & & & -3.66 & $* 1.63$ & & & -4.36 & $* 0.76$ \\
\hline $\begin{array}{l}\text { Craft, rela. } \\
\text { Trades }\end{array}$ & & & -2.76 & $* 0.49$ & & & -2.23 & $* 0.43$ & & & -2.70 & $* 0.53$ \\
\hline Plant, machine & & & -4.57 & $* 0.42$ & & & -4.37 & $* 0.43$ & & & -5.17 & $* 0.51$ \\
\hline
\end{tabular}


Table 2 (continued)

\begin{tabular}{|c|c|c|c|c|c|c|c|c|c|c|c|c|}
\hline \multirow{2}{*}{$\begin{array}{l}\text { Explanatory } \\
\text { variables }\end{array}$} & \multicolumn{2}{|l|}{ M1 } & \multicolumn{2}{|l|}{ M2 } & \multicolumn{2}{|l|}{ M1 } & \multicolumn{2}{|l|}{ M2 } & \multicolumn{2}{|l|}{ M1 } & \multicolumn{2}{|l|}{ M2 } \\
\hline & $\mathrm{C}$ & SE & $\mathrm{C}$ & SE & $\mathrm{C}$ & $\mathrm{SE}$ & $\mathrm{C}$ & SE & $\mathrm{C}$ & SE & $\mathrm{C}$ & SE \\
\hline Elementary & & & -5.18 & $* 0.49$ & & & -5.39 & $* 0.52$ & & & -4.89 & $* 0.54$ \\
\hline Unknown & & & & & & & 5.19 & $* 0.64$ & & & -4.83 & $* 1.37$ \\
\hline \multirow[t]{2}{*}{$\mathrm{N}$} & \multicolumn{4}{|c|}{2572} & \multicolumn{4}{|c|}{2544} & \multicolumn{4}{|c|}{2543} \\
\hline & \multicolumn{4}{|c|}{ Sweden } & \multicolumn{4}{|c|}{ Turkey } & \multicolumn{4}{|c|}{ United Kingdom } \\
\hline Aged 20-29 & -0.63 & $* 0.24$ & 0.53 & $* 0.25$ & 0.92 & 0.50 & 0.93 & $* 0.46$ & 0.25 & 0.26 & 0.63 & $* 0.26$ \\
\hline Aged 40-49 & -0.53 & $* 0.23$ & -0.97 & $* 0.32$ & -0.40 & 0.36 & 0.40 & 0.37 & -0.68 & $* 0.28$ & -0.30 & 0.30 \\
\hline Aged 50-59 & -0.68 & $* 0.25$ & -1.33 & $* 0.48$ & -1.44 & $* 0.62$ & -0.12 & 0.64 & -1.73 & $* 0.29$ & -0.58 & 0.43 \\
\hline $\begin{array}{l}\text { Low. secon. } \\
\text { Less }\end{array}$ & & & -0.19 & 0.32 & & & -0.95 & $* 0.39$ & & & -1.02 & $* 0.36$ \\
\hline $\begin{array}{l}\text { Higher } \\
\text { education }\end{array}$ & & & -0.08 & 0.19 & & & 0.81 & 0.43 & & & 0.65 & $* 0.22$ \\
\hline $\begin{array}{l}\text { Unkn. educ. } \\
\text { Lev. }\end{array}$ & & & & & & & & & & & -0.35 & 0.99 \\
\hline Skills level & & & -0.01 & $* 0.00$ & & & 0.01 & $* 0.00$ & & & -0.00 & 0.00 \\
\hline \multicolumn{13}{|l|}{ Occupation } \\
\hline Armed forces & & & 0.79 & 1.16 & & & 2.03 & 2.09 & & & 0.93 & 0.95 \\
\hline Managers & & & 0.26 & 0.30 & & & 0.86 & 0.64 & & & 0.24 & 0.29 \\
\hline $\begin{array}{l}\text { Techn., ass. } \\
\text { Prof. }\end{array}$ & & & -0.03 & 0.22 & & & -0.39 & 0.65 & & & 0.29 & 0.26 \\
\hline Clerical support & & & -1.93 & $* 0.44$ & & & -1.69 & $* 0.81$ & & & -1.30 & $* 0.33$ \\
\hline $\begin{array}{l}\text { Service and } \\
\text { sales }\end{array}$ & & & -1.70 & $* 0.25$ & & & -2.08 & $* 0.80$ & & & -0.42 & 0.28 \\
\hline $\begin{array}{l}\text { Agricul., } \\
\text { forestry }\end{array}$ & & & -2.57 & $* 1.18$ & & & -5.57 & $* 0.89$ & & & -3.21 & 2.34 \\
\hline $\begin{array}{l}\text { Craft, rela. } \\
\text { Trades }\end{array}$ & & & -1.83 & $* 0.40$ & & & -2.34 & $* 0.78$ & & & -1.06 & 0.58 \\
\hline Plant, machine & & & -3.71 & $* 0.35$ & & & -4.19 & $* 0.82$ & & & -3.96 & $* 0.52$ \\
\hline Elementary & & & -4.92 & $* 0.59$ & & & -4.13 & $* 0.78$ & & & -3.92 & $* 0.43$ \\
\hline Unknown & & & 0.31 & 0.67 & & & 1.62 & 2.70 & & & -0.57 & 1.34 \\
\hline $\mathrm{N}$ & \multicolumn{4}{|c|}{2570} & \multicolumn{4}{|c|}{1543} & \multicolumn{4}{|c|}{4363} \\
\hline
\end{tabular}

1) The table shows the estimated coefficients (C) and standard errors (SE) for each country, where we use linear regression (constant terms included). 2) * Significant at the 5\% level

The final sample consists of 75,777 employees living in 27 European and nonEuropean countries. The sample size $(\mathrm{N})$ by country is shown in Tables 1 and 2 . The data for the Russian Federation only covers the Moscow municipal area.

\section{The Dependent Variable}

The dependent variable measures IPP. IPP is measured by the following five individual characteristics: (1) how often a person learns new work-related things from co-workers or supervisors in his or her own job (abbreviated formulation 'learning new work- 
related things'), (2) how often a person's job involves learning-by-doing from the tasks he or she performs ('learning-by-doing from tasks'), (3) how often a person's job involves keeping up to date with new products ('keeping up to date with new products'), (4) how often a person's job usually involves instructing, training or teaching people, individually or in groups ('instructing, training or teaching people'), and (5) how often a person's job usually involves advising people ('advising people').

For each of the characteristics (1)-(5), the response categories are: 1 Never, 2 Less than once a month, 3 Less than once a week but at least once a month, 4 At least once a week but not every day, and 5 Every day. Since the Cronbach's alpha for the five characteristics is within the acceptable range for the internal consistency of these characteristics (i.e. alpha $>0.7$ ) for all countries as a whole, we include them in the same scale as an overall measure of IPP and treat this measure as one-dimensional.

We have also carried out a (principal-component) factor analysis for all countries as a whole. After orthogonal rotation (varimax), this factor analysis gives us a twovariable factor-solution, but prior to rotation the factor-solution shows that each of the five items in the measure of IPP has a higher factor loading on the first factor variable than on the second factor variable, which in addition to the alpha-score supports our choice of including all five items into one single variable. The dependent variable is therefore set equal to the sum of the characteristics (1)-(5), and thus varies from 5 to 25 .

\section{The Explanatory Variables}

Endogeneity is a common problem in regression analyses, which refers here to the possibility that the values of at least one of the explanatory variables is affected by the dependent variable. Instrumental variables (IV) techniques are commonly used to address this problem. According to Chang and Kang (2018), the instruments must be both relevant and exogenous. They suggest strategies to test both the relevance and exogeneity requirements. It is likely that at least one of several potential instruments will be related to an explanatory variable that we suspect to be endogenous. Therefore, we will probably be able to find an instrument that is associated with the variable that may be endogenous. However, the problem is to find exogenous instruments; that is, they should not be affected by the employees' IPP, but correlated with the explanatory variable suspected to be endogenous. Based on the PIAAC data set, we have no suggestions for such instruments. Furthermore, the cross-sectional nature of the PIAAC data prevents us from lagging variables that we suspect to be endogenous, and using the lagged variables as instruments to reduce the potential endogeneity of the variables that are lagged. Because it is difficult to find suitable instruments, we cannot use IV techniques in the analysis. As a substitute for this, we try to reduce the potential bias from endogeneity by not controlling for individual characteristics that we expect will be affected by the measure of IPP (for example, characteristics like 'confronting more complex problems', and 'how often the job usually involves planning own activities').

We have also selected the explanatory variables to account for the potential multicollinearity problem. This selection is based on calculations of the Variance Inflation Factor (VIF). These calculations indicate that we have no serious multicollinearity problems. 
In accordance with the purpose of the article, age is the only key regressor in the analysis. Since we lack information about exact age for some of the countries, we use four age dummy variables in the regressions: 20-29 years, 30-39 years (the reference category), 40-49 years, and 50-59 years.

The following control variables are included in the estimations: gender ( $1=$ female, $0=$ male), educational level (four dummy variables), skills level, seniority, participation in formal and non-formal learning, weekly working hours, firm size (six dummy variables), occupation (11 dummy variables), and industrial sector affiliation (12 dummy variables). Controlling for occupation accounts to some degree for characteristics that are more relevant for specific jobs such as physical strength and innovative learning.

There are three educational levels: lower secondary or less, upper secondary or postsecondary (the reference category), and higher education. We also include a category for those with unknown educational level.

We have chosen to measure skills level as literacy skills, since the correlation between literacy skills and numeracy skills is very high. ${ }^{2}$ Literacy (and numeracy) skills in the PIAAC database consist of a set of ten plausible values estimated for each person.

Seniority measures approximately how many years a person has had paid work in total (only years where 6 months or more was spent in either full-time or part-time work). We control for seniority since age is highly correlated with seniority for a particular occupation, ${ }^{3}$ and since employees in different occupations may begin their active careers at different ages. The age variables are thus net of seniority effects.

We differentiate between participation in formal and non-formal learning. Participants in formal learning are studying for any kind of formal qualification at the time of the interview, or have studied for any formal qualification during the last 12 months. Participants in non-formal learning have participated in at least one of the following activities during the last 12 months: open or distance education, on-the-job training or training by supervisors or co-workers, seminars or workshops, and other courses or private lessons.

Firm size (i.e. number of employees) is a variable with five categories: 1-10 employees, 11-50 employees, 51-250 employees (the reference category), 251-1000 employees, and more than 1000 employees. We also include a category for those with unknown firm size.

Occupation is based on ISCO codes (1-digit), where 'professionals' is used as the reference category. Persons with a manual occupation are either employed as 'clerical support workers', 'service and sales workers', 'skilled agricultural, forestry and fishery workers', 'craft and related trades workers', 'plant and machine operators, and assemblers', or employed in 'elementary occupations'. Industrial sector is based on ISIC

\footnotetext{
${ }^{2}$ The pairwise correlation coefficient between literacy and numeracy skills is 0.90 for the 27 countries as a whole.

${ }^{3}$ The pairwise correlation coefficient between exact age and seniority for a particular occupation is in the interval $[0.76,0.93]$ for the 25 countries in the sample as a whole with non-missing values for exact age (except New Zealand, Singapore). The corresponding coefficients between the age dummy variables and seniority for the 27 countries as a whole are much lower (measured in absolute value): $[-0.72,-0.48]$ (20-29 years), $[-0.48$,$0.14]$ (30-39 years), [0.13,0.53] (40-49 years), and [0.53,0.69] (50-59 years).
} 
codes (alphabetical level), where 'trade, transportation and service' is used as the reference category.

Skills level, seniority and weekly working hours are continuous variables. All other explanatory variables are dummy variables.

\section{The Weighting Procedures}

All results in Table 2 and Fig. 1 are weighted by using the full sample (final) weight and the 80 replicate weights in the PIAAC database. The weighting procedure ensures representative data. The 'repest' command in Stata, which is used when weighting the results, ensures correct estimates for standard errors for the skills level.

This procedure is also used when weighting the results in Table 3, but in this case the weights are corrected to provide that the number of observations for all the 27 countries is the same. We set this number to 5200 in each country (see Støren 2015, Appendix 1). This weighting procedure secures that all the country samples will have the same influence on the results when using all countries as a whole.

In Table 4, the results are weighted by using the 'mixed' command in Stata, where we only use the full sample (final) weight (based on sampling weighting). Sampling weights are specified at the first level in the multilevel model. The type of standard error reported is derived from asymptotic theory. A likelihood-ratio test is carried out after the regression (in M6). Since this test is invalid in the case of sampling weighted estimators, the test is based on frequency weighting. In this case, Stata produces exactly the same results (in terms of estimated coefficients and standard errors, including

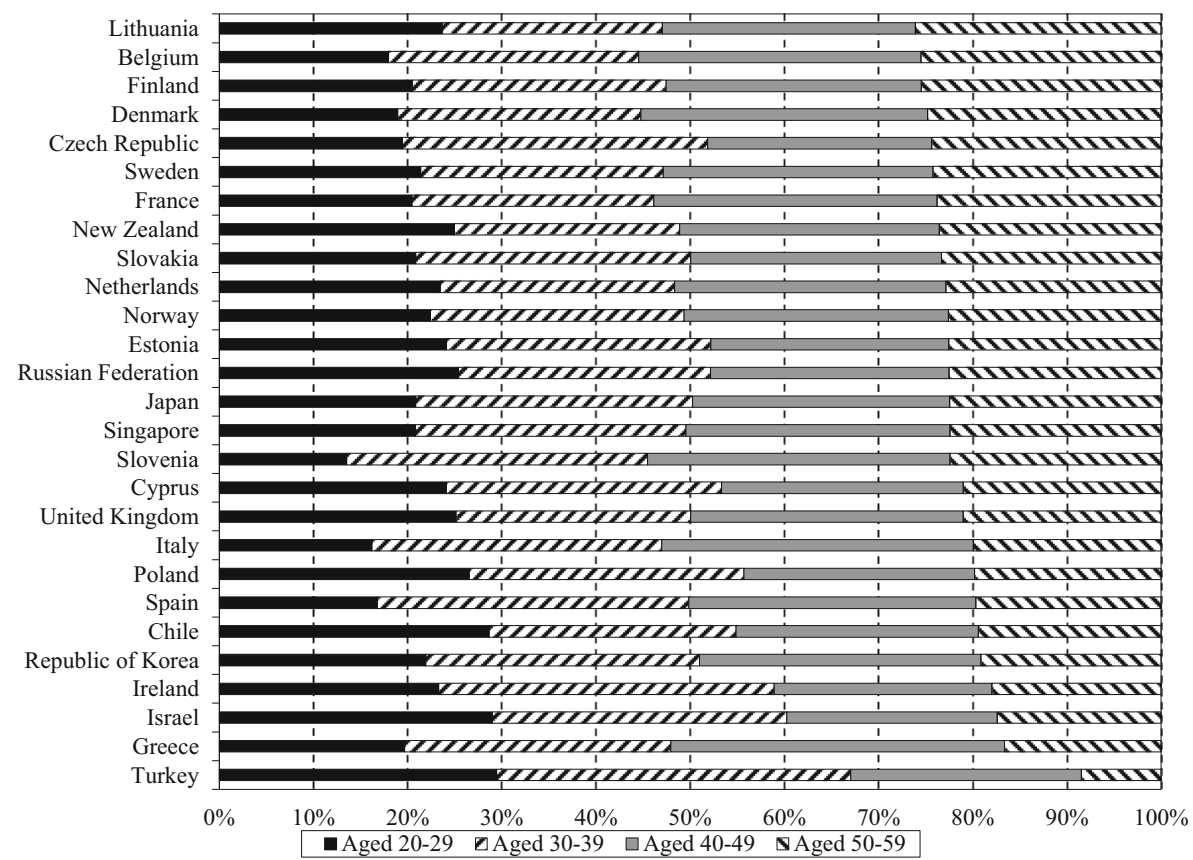

Fig. 1 Composition of sample of employees, by country and age 
Table 3 Estimated effects on the country-specific estimated coefficient for the oldest age group (M3) and the probability that this estimated coefficient is significant (M4), all countries as a whole

\begin{tabular}{|c|c|c|c|c|}
\hline \multirow[t]{2}{*}{ Explanatory variables } & \multicolumn{2}{|l|}{ M3 } & \multicolumn{2}{|l|}{ M4 } \\
\hline & $\mathrm{C}$ & SE & $\mathrm{C}$ & SE \\
\hline \multicolumn{5}{|l|}{ Individual level } \\
\hline IPP score & -0.00 & $* 0.00$ & 0.01 & $* 0.00$ \\
\hline Lower secondary or less & -0.07 & $* 0.01$ & 0.39 & $* 0.04$ \\
\hline Higher education & -0.02 & $* 0.01$ & 0.10 & $* 0.03$ \\
\hline Unknown educational level & 0.31 & $* 0.12$ & -1.61 & $* 0.41$ \\
\hline Skills level & -0.00 & $* 0.00$ & 0.00 & $* 0.00$ \\
\hline \multicolumn{5}{|l|}{ Occupation } \\
\hline Armed forces occupations & -0.11 & $* 0.04$ & 0.51 & $* 0.15$ \\
\hline Managers & 0.11 & $* 0.01$ & -0.41 & $* 0.05$ \\
\hline Technicians and associate professionals & 0.04 & $* 0.01$ & -0.17 & $* 0.04$ \\
\hline Clerical support workers & 0.06 & $* 0.01$ & -0.35 & $* 0.06$ \\
\hline Service and sales workers & 0.03 & $* 0.01$ & -0.14 & $* 0.05$ \\
\hline Skilled agricultural, forestry and fishery workers & 0.06 & 0.04 & -0.48 & $* 0.16$ \\
\hline Craft and related trades workers & 0.01 & 0.01 & -0.09 & 0.06 \\
\hline Plant and machine operators, and assemblers & 0.03 & 0.02 & -0.24 & $* 0.07$ \\
\hline Elementary occupations & 0.04 & $* 0.01$ & -0.26 & $* 0.07$ \\
\hline Unknown occupation & 0.26 & $* 0.02$ & 0.23 & $* 0.11$ \\
\hline \multicolumn{5}{|l|}{ National level } \\
\hline Average IPP score & 0.03 & $* 0.00$ & 0.38 & $* 0.01$ \\
\hline Average skill loss for the oldest age group & -0.00 & $* 0.00$ & 0.06 & $* 0.00$ \\
\hline Average skills level for all age groups & -0.01 & $* 0.00$ & 0.07 & $* 0.00$ \\
\hline Proportion of employees with higher education & -0.00 & $* 0.00$ & -0.10 & $* 0.00$ \\
\hline $\mathrm{N}$ & \multicolumn{4}{|c|}{75,777} \\
\hline
\end{tabular}

1) The table shows the estimated coefficients (C) and standard errors (SE) for all countries as a whole, where we use linear regression in M3 and logistic regression in M4 (constant terms included). 2) In M3, the dependent variable is the estimated coefficients for the oldest age group in Table 2 (M2). 3) In M4, the dependent variable is equal to 1 if an estimated coefficient for the oldest age group in Table 2 (M2) is significant (at the $5 \%$ level), and 0 otherwise. 4 ) * Significant at the $5 \%$ level

estimates of random-effects parameters), regardless of whether we use sampling or frequency weighting.

All calculations of the VIFs are weighted, where we only use the full sample (final) weight (based on sampling weighting). The results from the factor analysis in "The Dependent Variable" section, and the calculations of the pairwise correlation coefficients in "The Explanatory Variables" section, are also weighted by using only the full sample weight (based on analytic weighting). The Cronbach's alpha calculation in "The Dependent Variable" section, and the calculations of the pairwise correlation coefficients in "Descriptive Statistics" and "The Effects of National Averages" sections, are done without using any weights. 
Table 4 Estimated effects on the IPP score, all countries as a whole

\begin{tabular}{|c|c|c|c|c|}
\hline \multirow[t]{2}{*}{ Explanatory variables } & \multicolumn{2}{|l|}{ M5 } & \multicolumn{2}{|l|}{ M6 } \\
\hline & $\mathrm{C}$ & SE & $\mathrm{C}$ & $\mathrm{SE}$ \\
\hline \multicolumn{5}{|l|}{ Individual level } \\
\hline Aged 20-29 & 0.01 & 0.05 & 0.49 & $* 0.05$ \\
\hline Aged $40-49$ & -0.68 & $* 0.05$ & -0.54 & $* 0.05$ \\
\hline Aged $50-59$ & -1.39 & $* 0.05$ & -1.05 & $* 0.07$ \\
\hline Lower secondary or less & & & -0.74 & $* 0.05$ \\
\hline Higher education & & & 0.41 & $* 0.09$ \\
\hline Unknown educational level & & & 0.12 & 0.65 \\
\hline Skills level & & & -0.00 & 0.00 \\
\hline \multicolumn{5}{|l|}{ Occupation } \\
\hline Armed forces occupations & & & 0.53 & $* 0.21$ \\
\hline Managers & & & 0.61 & $* 0.07$ \\
\hline Technicians and associate professionals & & & -0.50 & $* 0.06$ \\
\hline Clerical support workers & & & -1.83 & $* 0.07$ \\
\hline Service and sales workers & & & -1.20 & $* 0.06$ \\
\hline Skilled agricultural, forestry and fishery workers & & & -3.24 & $* 0.20$ \\
\hline Craft and related trades workers & & & -2.07 & $* 0.08$ \\
\hline Plant and machine operators, and assemblers & & & -3.89 & $* 0.08$ \\
\hline Elementary occupations & & & -4.43 & $* 0.08$ \\
\hline \multirow[t]{2}{*}{ Unknown occupation } & & & -1.01 & $* 0.19$ \\
\hline & & & E & SE \\
\hline \multicolumn{5}{|l|}{ National level } \\
\hline Skills level & & & 0.17 & 0.06 \\
\hline Higher education & & & 0.00 & 0.00 \\
\hline $\mathrm{N}$ & \multicolumn{4}{|c|}{75,777} \\
\hline
\end{tabular}

1) The table shows the estimated coefficients (C), estimates (E) and standard errors (SE) for all countries as a whole, where we use multilevel mixed-effects linear regression (constant terms included). 2) * Significant at the $5 \%$ level

\section{The Econometric Methods}

Several econometric methods are used in the analysis. When examining whether the effects of the explanatory variables on the IPP score differ between the countries, we run regressions for the 27 countries separately. One possibility is to use ordered logistic regression in this case. The problem is that the assumptions of the ordered logit model are often violated (Williams 2016). We therefore prefer to use linear regression, for two further reasons. First, since the dependent variable can be seen as a coarsened version of a continuous variable, and not a truly ordered variable, we can derive a meaningful scale of the dependent variable. Second, the results from linear regression are in most cases easier to interpret than the corresponding results from ordered logistic 
regression. ${ }^{4}$ Based on linear regression, larger values of the dependent variable correspond to higher IPP scores.

We also run a regression for all countries as a whole using multilevel mixed-effects linear regression. In this case, we examine the effects of the explanatory variables on the IPP score at the individual level and the effects of some of these variables (i.e. the 'higher education' variable and the skills level) at the national level.

Two further regressions are performed for all countries as a whole. One is based on linear regression, where we examine the effects of explanatory variables on the country-specific estimated coefficient for the oldest age group. The other is based on logistic regression, where we examine the effects of the same explanatory variables on the probability that this estimated coefficient is significant (at the $5 \%$ level).

\section{Descriptive Statistics}

Figure 1 presents the distribution of age by country. We see that Lithuania (26\%), Belgium (25\%), Finland (25\%) and Denmark (25\%) have the highest proportions of the oldest age group, while Turkey (8\%), Greece (17\%) and Israel (17\%) have the lowest. As a consequence of these results, Turkey has the highest proportions of those aged 30 $39(38 \%)$ and those aged 20-29 (29\%). Lithuania (23\%) is found to have the lowest proportion of those aged 30-39, while Slovenia (14\%) has the lowest proportion of the youngest age group. Although Greece and Israel have the same proportions of the oldest age group, Greece (35\%) has the highest proportion and Israel $(22 \%)$ the lowest proportion of those aged 40-49.

Table 1 presents the following four national averages by country: the average IPP score, the average skill loss for the oldest age group, the average skills level for all age groups, and the proportion of employees with higher education. The average skill loss for the oldest age group is measured as the difference in the average (literacy) skills level between the youngest age group (aged 20-29) and the oldest age group (aged 5059) divided by the average of the standard deviations for these two age groups (multiplied by 100). The proportion of employees with higher education (at the national level) is measured in percent.

These four variables at the national level are not found to be significantly related to each other (at the 5\% level), based on pairwise correlation coefficients between the variables where country is the unit of observation. The strongest pairwise correlation coefficient is between the average skills level for all age groups and the proportion of employees with higher education (0.36), but the estimated coefficient is only significant at the $10 \%$ level.

We find from Table 1 that New Zealand, Norway, Finland and Sweden have the highest average IPP scores, while Lithuania, the Republic of Korea and Turkey have the lowest. The average skill loss for the oldest age group is highest in Singapore and the Republic of Korea, and lowest in the Russian Federation and Cyprus.

\footnotetext{
${ }^{4}$ Hellevik (2009) argues that 'the statistical arguments against the use of linear regression with a binary dependent variable are not as decisive as it is often claimed... The results for linear and logistic significance probabilities as we have seen turn out to be nearly identical, even with small samples and skewed distributions on the dependent variable... This means that we are not restricted to use logistic regression with a binary dependent variable' (p. 73).
} 
The table shows that Japan, Finland, the Netherlands and Sweden have the highest average skills levels, while Chile, Turkey, Italy and Greece have the lowest levels. The countries of Northern Europe have much higher average skills levels than the countries of Southern Europe.

The Russian Federation (70\%), Singapore (58\%) and Israel (53\%) have the highest proportions of employees with higher education, while Italy (17\%), the Czech Republic (22\%), Slovakia (26\%) and Turkey (27\%) have the lowest proportions. The result for the Russian Federation must be seen in the context that the data for this country only covers the Moscow municipal area.

\section{The Estimation Results}

The estimation results for each country are presented in Table 2. M1 shows the relationship between the age of the employees and the IPP score in each of the 27 countries. M2 shows this relationship when we include the control variables. Due to space limitations, the table only shows the estimated effects of age, educational level, skills level and occupation, but we also comment on some of the other control variables.

\section{The Effects of the Key Regressor}

M1 shows that only two of the 27 countries (Chile, Spain) have non-significant effects of the oldest age group on the IPP score (at the 5\% level). In all the other countries, the oldest employees have a significantly lower score than the reference group (aged 3039). The hypothesis H1 is therefore supported for 25 of the 27 countries, but the form of the bivariate relation between age and IPP, as well as the size of it, varies between the countries.

Only two countries have a clear age gradient in the IPP score (France, Lithuania), which means that the score declines successively with increasing age group. Furthermore, it is considerable variation in the age effects between the countries. Ireland, the Republic of Korea, the Russian Federation and Singapore have 2-3 points lower IPP score among the oldest employees than among those in the reference group. In the four Nordic countries, Japan and New Zealand, this difference is less than one point, even though it is statistically significant in each of these countries.

When the control variables are introduced (M2), the number of countries with nonsignificant age differences between the oldest employees and those in the reference group increases from two to 10 countries (Belgium, Chile, Cyprus, the Czech Republic, Greece, Israel, New Zealand, the Russian Federation, Turkey, the UK). The IPP score among 50-59 year olds is found to be significantly lower than among 30-39 year olds in all the remaining 17 countries. In 14 of the 17 countries with significant age effects in M2 (except Estonia, Norway, Poland) there is a reduction of more than one point in the IPP score between employees aged 50-59 and the reference group. Seven countries (Finland, France, Lithuania, the Netherlands, Slovenia, Spain, Sweden) have a clear age gradient in the IPP score.

We also see from Table 2 that there is a significant age effect for the oldest age group in both M1 and M2 for 16 countries. The question is then whether the change in 
the age effect between M2 and M1 for these 16 countries is significant? We find that the age differences are reduced in eight of the 16 countries (Estonia, France, Ireland, the Republic of Korea, Lithuania, Poland, Singapore, Slovakia) and increased in the remaining eight other countries, when the control variables are included. Except for four countries (Estonia, the Republic of Korea, Poland, Singapore), none of the other reductions in the age effects on the IPP score are significant (based on a 95\% CI). This means that the age effect for the oldest age group seems to be statistically robust in most of the 16 countries, in the sense that it is not particularly influenced by the inclusion of the control variables. Thus, the hypothesis H4a is not supported for most of the countries, but it is supported for four countries.

\section{The Effects of the Control Variables}

Table 2 shows that the educational level has a non-significant effect on the IPP score in five of the countries (Chile, Greece, New Zealand, Poland, Sweden), while one country (Japan) has only a significant (positive) effect of those with unknown educational level. There is a clear educational gradient in the IPP score in five of the other 21 countries (Belgium, Denmark, Estonia, Italy, the UK). In five other countries (Cyprus, the Czech Republic, Ireland, Lithuania, the Russian Federation), employees with higher education have a higher IPP score than the reference group. In the remaining 11 countries, employees with a low educational level have a lower score than the reference group. This means that in most of the countries, formal education increases the IPP score significantly. When we add that skills are not significantly associated with the score in 19 of the countries, and that the significant effects are quite modest and are most often negative (only positive for Spain and Turkey), these results support the hypothesis $\mathrm{H} 2(\mathrm{a})$, while the hypotheses $\mathrm{H} 2$ (b) and $\mathrm{H} 3$ are not supported. Thus, the confidence in a human capital interpretation is strengthened at the expense of the signalling theory's understanding of how such variables should be related to each other. Small negative effects of skills on the IPP score may also indicate that some people with a relatively low skills level try to compensate for this by increasing their skills utilisation at work.

In more than half of the countries, all employees with a manual occupation have a lower IPP score than professionals (the reference group), and being employed in at least one of the manual occupations gives a relatively lower score in all countries. The difference is quite large, up to 2-7 points on the IPP scale in each country based on those manual occupations where there is a significant (negative) effect. No employees with a manual occupation have a significantly higher IPP score than professionals. These results indicate that Goldthorpe's (2007) distinction between service and labour contracts sheds light on important differences in the IPP score between different groups of workers. Furthermore, we find that there are differences in the IPP score in different industries. The reference category 'trade, transportation and service' has a higher score than 'agriculture, forestry, fishing', 'mining, manufacturing, electricity', 'professional, scientific' and 'public, defence' in many countries. We therefore conclude that the hypothesis $\mathrm{H} 4 \mathrm{~b}$ is quite strongly supported in M2.

There is a clear tendency for full-time workers to have a higher IPP score than those who work part-time. The variable measuring weekly working hours is positive and significant for almost all countries (only a non-significant effect for Chile). 
Females have a lower IPP score than males in Belgium, Chile, Cyprus, Estonia, Japan, the Republic of Korea, Poland, Singapore and Spain. All the other 18 countries have non-significant gender differences in the IPP score (at the 5\% level). For most countries we find that the effect of seniority on the IPP score is non-significant, while for some countries this variable has a positive effect (Denmark, Finland, Ireland, Italy, the Netherlands, Norway, Slovenia, Spain).

The IPP score clearly correlates with participation in non-formal learning. In all countries, there is a positive relationship. The score rarely correlates with participation in formal learning and the firm size variables.

\section{The Effects of National Averages}

Now, we ask whether the country-specific estimated coefficients for the oldest age group in Table 2 (M2) are related to the aggregated characteristics of the 27 countries (in Table 1). Table 3 shows the effects of individual characteristics and the four averages at the national level on the estimated coefficient for the oldest age group in a country (M3), and the probability that this estimated coefficient is significant (M4), where we use all countries as a whole. The explanatory variables at the individual level are the same as in Table 2 (M2), except that the age dummy variables are replaced with the individual IPP score. VIF calculations based on the linear regression in Table 3 indicate that there are no serious multicollinearity problems. ${ }^{5}$

We see from Table 3 that there is a positive relationship between the estimated coefficient for the oldest age group in a country and the average IPP score, and a negative relationship for the three other averages at the national level. The proportion of employees with higher education has a negative effect on the probability that the estimated coefficient for the oldest age group is significant, while each of the three other national averages has a positive effect. At the individual level, we find from the table that the estimated coefficient for the oldest age group is negatively related to the IPP score, the skills level, and the 'lower secondary or less' and 'higher education' categories. Each of these four individual characteristics is positively related to the probability that the estimated coefficient is significant. Most of the manual occupations are negatively related to this probability, while half of the manual occupations are positively related to the estimated coefficient for the oldest age group.

In Table 4, we examine how the IPP score is affected by the age dummy variables when we exclude (M5) and include (M6) the control variables. M6 also includes two explanatory variables (the 'higher education' variable and the skills level) at the national level. The results in the table are based on multilevel mixed-effects linear regression, where we use all countries as a whole. The dependent variable and the explanatory variables at the individual level in M5 and M6 are the same as in M1 and M2 in Table 2, respectively. VIF calculations based on linear regression (using the variables at the individual level in Table 4) indicate that we have no serious multicollinearity problems.

\footnotetext{
${ }^{5}$ We have estimated the pairwise correlation coefficient between each of the four national-level variables in Table 1 and the country-specific estimated coefficients for the oldest age group, where country is the unit of observation, but none of the estimated coefficients are significant at the $10 \%$ per cent level.
} 
Table 4 shows that the oldest employees have significantly lower IPP score than the middle-aged employees in both M5 and M6, but there is only a clear age gradient in the score in M6. Employees with higher education have a higher IPP score than the reference group, while employees with a low educational level have a relatively lower score. The IPP score is not significantly affected by skills at the individual level. All employees with a manual occupation have a lower IPP score than professionals, and the difference is 2-4 points on the score for most of these occupations. At the national level, the skills level and the 'higher education' variable have positive effects on the IPP score, and it seems that these effects are significant given a 95\% CI for each of the two national-level variables and based on a likelihood-ratio test after the regression.

It follows from these results that countries with significant age differences in the IPP score between the middle-aged and the oldest workers have a larger average skill loss for the oldest age group and/or a larger average skills level for all age groups at the national level than countries without such significant age differences (on average), after controlling for several individual characteristics and national differences in the average IPP score and the proportion of employees with higher education. This indicates that it is most demanding for the oldest workers to keep up with the IPP of younger workers where the national averages in skill loss or skills level (or both) are highest.

\section{Discussions and Conclusions}

We find that the oldest employees have a lower IPP score than the middle-aged employees for 25 of the 27 countries, when the control variables are not included. Thus, the answer to the question whether age is associated with a significant reduction in activities which promote or enhance potential productivity is yes, it does so in nearly all the 27 countries. Basically, we tend to link this to the employer's allocation of work tasks in the workplace. Introducing the control variables in the regressions, we find that the oldest employees have a lower IPP score than the middle-aged employees for 17 of the 27 countries, while for the remaining 10 countries we find no significant differences in this score between the oldest and middle-aged employees. The age effect for the oldest age group on IPP is not particularly influenced by the inclusion of the control variables in the majority of the countries, and therefore seems to be statistically robust. In countries with significant age effect (for the oldest age group) in M1 and nonsignificant age effect in M2, control variables with significant effects on IPP act as mediators for age differences in IPP.

Formal education has significant positive impact on IPP in most countries. Thus, even though we account for documented cognitive skills, variation in IPP is still explained by formal education. Since we use occupation and industry as control variables and thus take into account the possible gateway effects associated with educational segmentation and credentialism in the labour market, significant positive effects of formal education and the absence of significant effects of skills on the IPP score in many of the countries, are easier to adapt to the human capital perspective on the importance of formal education than to the competing perspective of the signalling theory. According to the signalling theory, cognitive skills should bear the explanatory power of the model, not formal education. Since we study established employment relations, we assume that the employer has this relevant information (it is not an 
information problem), and apparently to a greater extent allocates learning and innovative work tasks according to formal education, occupation and age than by cognitive skills.

Although skills have hardly any direct impact on the IPP score at the individual level, observed differences in the average skills level between the youngest and oldest employees (i.e. so-called skill loss for the oldest age group) and the average skills level for all age groups are important at the national level. It appears that it is a major challenge for the oldest workers to keep up with the IPP of younger workers if the average skill loss for the oldest age group and the average skills level in a country are high (or at least on one of these national averages). An alternative interpretation is that the allocation of such potentially productive work tasks reduces the older employees' opportunity to keep up with the potential productivity of younger employees in countries which is characterised by a high average skills level in the workforce. This seems to be more prevalent in Northern European countries and Japan where the general skills level is high than in Southern European countries where the general skills level is lower.

The IPP score is clearly influenced by the employee's professional status, which is in line with Goldthorpe's (2007) distinction between 'service contracts' and 'labour contracts'. Then we have an empirically rooted argument that Lazear's theory should differentiate between different positions in the labour market, but it may still be the case that both firms and employees would benefit from the fact that the working life is structured according to Lazear's general theory. There is also a clear relationship between the IPP score and participation in non-formal learning. These two findings point to a possible focus area for individual businesses, industries and the government: firms should involve more groups of employees, especially employees with a manual occupation and those with a low participation rate in non-formal learning, since there is a clear tendency for professionals to have a higher IPP score than workers with a manual occupation, and that participation in non-formal learning positively correlates with this score. Furthermore, the fact that formal education influences the IPP score, is a fairly strong indication that access to formal education when somewhat younger is probably important for the IPP of older employees, and this should primarily be considered as a public authority task. Here we allow ourselves to dynamise the interpretation of a finding that is based on observations at one point in time (a crosssectional view). In many European industrialised countries, efforts are now being made to increase the population's skills through formal education, and this policy also includes middle-aged employees and job seekers through measures of qualification and lifelong learning.

On the basis of these results, we can conclude that especially the formal education and occupation of the employee mediate age differences in 10 of the 27 countries (i.e. function as 'mechanisms'), while these hallmarks operate as additional explanations for differences in IPP in the remaining 16 countries. Chile has no significant age differences in IPP in any of the models M1 and M2. At the individual level, cognitive skills are not associated with potential productivity, but the average skills level (and skill loss due to age) is significantly associated with the average reduction of IPP due to age at the national level. Japan and Northern European countries have the highest average skills level in the PIAAC sample. This is our contribution to a theory of how various 
aspects of human capital are related to activities that promote potential productivity (IPP) at work (cf. Skirbekk 2004, 2008).

Two important reservations of our study must be made. First, the sample only includes employees. Thus, there may be a selection problem in the analysis, since the average employment rate varies a lot between different PIAAC countries (Grøgaard and Børing 2017). This rate will also vary for a particular age group between countries, including older workers. For example, there are differences in pension behaviour and the right to retire between countries. A country's employment rate may also be related to the average educational level in the country. By setting the upper limit for age at 59, we have reduced the problem related to early retirement in some countries without removing it completely.

We have tried to examine whether we have a selection problem in the analysis. This is done by estimating the pairwise correlation coefficient between the country-specific estimated coefficients for the oldest age group and each of the following five nationallevel variables, where country is the unit of observation: the average employment level among persons in the 20-29 age group, 30-39 age group, 40-49 age group, 50-59 age group, and 20-59 age group. Persons include those who are employed, unemployed and outside the labour force. Based on the PIAAC data, we find that none of the estimated coefficients are significant at the $10 \%$ per cent level. Thus, these estimations indicate that we have no serious selection problem.

Secondly, in a cross-sectional survey, like PIAAC, we do not know what kind of skills and IPP the elderly had when they were younger. Estimated age effects can therefore express generation effects and not life-phase effects (see Desjardins and Warnke 2012 for a discussion of the relationship between ageing and skills). In order to examine whether it is a life-phase effect or a generational effect, we need in principle a panel design. Thus, there is a clear need for more evidence of the relationship between employees' age and their IPP.

Acknowledgements The authors thank anonymous referees for helpful and constructive comments on earlier versions of this article. All remaining errors are our own.

Funding This work was funded by the Research Council of Norway as part of the project 'Silver Lining' grant 255210 .

Data Availability The data can be downloaded from the OECD's website: http:/www.oecd.org/skills/ piaac/.

\section{Compliance with Ethical Standards}

Declaration of Interest The authors declare no conflicts of interest.

Open Access This article is licensed under a Creative Commons Attribution 4.0 International License, which permits use, sharing, adaptation, distribution and reproduction in any medium or format, as long as you give appropriate credit to the original author(s) and the source, provide a link to the Creative Commons licence, and indicate if changes were made. The images or other third party material in this article are included in the article's Creative Commons licence, unless indicated otherwise in a credit line to the material. If material is not included in the article's Creative Commons licence and your intended use is not permitted by statutory 
regulation or exceeds the permitted use, you will need to obtain permission directly from the copyright holder. To view a copy of this licence, visit http://creativecommons.org/licenses/by/4.0/.

\section{References}

Aiyar, S., Ebeke, C., and Shao, X. (2016). The Impact of Workforce Aging on European Productivity, IMF Working Paper 16/238.

Arrow, K. J. (1973). Higher education as a filter. Journal of Public Economics, 2, 193-216.

Aubert, P., and Crépon, B. (2006), Age, wage and productivity: Firm-level evidence. A French version of this study has been published in Économie et Statistique, 2003, 368, 95-119.

Barthel, J. (2008). Can age discrimination be justified with a lower productivity of older workers? MPRA Paper, 14682.

Becker, G. S. (1962). Investment in Human Capital: A theoretical analysis. Journal of Political Economy, 70, 9-49.

Börsch-Supan, A., \& Weiss, M. (2016). Productivity and age: Evidence from work teams at the assembly line. The Journal of the Economics of Ageing, 7, 30-42.

Carlsson, M., \& Eriksson, S. (2019). Age discrimination in hiring decisions: Evidence from a field experiment in the labor market. Labour Economics, 59, 173-183.

Cataldi, A., Kampelmann, S., \& Rycx, F. (2012). Does it pay to be productive? The case of age groups. International Journal of Manpower, 33, 264-283.

Chang, J., \& Kang, Y. (2018). Instrumental variable estimates of the effect of management practices on firm performance in korean firms. Journal of Labor Research, 17, 1-20.

Collins, R. (1979). The credential society: An historical sociology of education and stratification. New York: Academic Press.

Desjardins, R., and Warnke, A. (2012). Ageing and skills: A review and analysis of skill gain and skill loss over the lifespan and over time. OECD Education Working Papers, 72.

Doeringer, P. B., and Piore, M. J. (1971), Internal Labor Markets and Manpower Analysis, Lexington, Mass., Heath.

Freeman, R. (1997). Are Norway's Solidaristic and welfare state policies viable in the modern global economy? In J. E. Dølvik \& A. Steen (Eds.), Making solidarity work? The Norwegian labour market model in transition (pp. 17-49). Oslo: Scandinavian University Press.

Göbel, C., \& Zwick, T. (2012). Age and productivity: Sector differences. De Economist, 160, 35-57.

Goldthorpe, J. H. (2007). 'Social class and the differentiation of employment contracts'. In: On Sociology, Volume II: Illustration and Retrospect, Second Edition, Stanford University Press, 101-124.

Grøgaard, J. B., and Børing, P. (2017). What matters most for adult employment-cognitive skills or formal education? Analysis of the selection to employment in 31 countries based on data from the PIAAC database, Report 2017:19, NIFU.

Hellerstein, J. K., and Neumark, D. (2004). Production function and wage equation estimation with heterogeneous labor: Evidence from a new matched employer-employee data set, NBER Working Paper 10325.

Hellevik, O. (2009). Linear versus logistic regression when the dependent variable is a dichotomy. Quality \& Quantity, 43, 59-74.

Kanfer, R., \& Ackerman, P. L. (2004). Aging, adult development, and work motivation. The Academy of Management Review, 29, 440-458.

Lallemand, T., \& Rycx, F. (2009). Are older workers harmful for firm productivity? De Economist, 157, 273292.

Lazear, E. P. (1979). Why is there mandatory retirement? Journal of Political Economy, 87, 1261-1284.

McEvoy, G. M., \& Cascio, W. F. (1989). Cumulative evidence of the relationship between employee age and job performance. Journal of Applied Psychology, 74, 11-17.

Mincer, J. (1958). Investment in Human Capital and Personal Income Distribution. Journal of Political Economy, 66, 281-302.

Moene, K. O., \& Wallerstein, M. (2003). Earnings inequality and welfare spending: A disaggregated analysis. World Politics, 55, 485-516.

Moene, K. O., and Wallerstein, M. (2005), The Scandinavian model and economic development, Special Report, Development Outreach, World Bank Institute. 
Neumark, D. (2020). Age discrimination in hiring: Evidence from age-blind vs. non-age-blind hiring procedures, NBER Working Paper 26623.

Neumark, D., \& Song, J. (2013). Do stronger age discrimination laws make social security reforms more effective? Journal of Public Economics, 108, 1-16.

$\mathrm{Ng}, \mathrm{T}$. W. H., \& Feldman, D. C. (2008). The relationship of age to ten dimensions of job performance. Journal of Applied Psychology, 93, 392-423.

OECD. (2013). Ageing and employment policies: Norway 2013: Working better with age. Paris: OECD Publishing.

OECD. (2016). Skills matter: Further results from the survey of adult skills, OECD Skills Studies. Paris: OECD Publishing.

Skirbekk, V. (2004). Age and individual productivity: A literature survey. Vienna Yearbook of Population Research, 2, 133-154.

Skirbekk, V. (2008). Age and productivity potential: A new approach based on ability levels and industrywide task demand. In A. Prskawetz, D. E. Bloom, \& W. Lutz (Eds.), Population aging, human capital accumulation, and productivity growth (pp. 191-207). New York: Population Council.

Solem, P. E. (2012). Possible effects of the financial crisis on managers' attitudes to older workers. Nordic Journal of Working Life Studies, 2, 129-142.

Spence, A. M. (1973). Job market signaling. Quarterly Journal of Economics, 87, 355-374.

Støren, L. A. (2015). Innovative learners at work: Results from the BRAIN project - The sub-project 'training, skills and innovation'. NIFU Report, 23.

Sturman, M. C. (2003). Searching for the inverted U-shaped relationship between time and performance: Meta-analyses of the experience/performance, tenure/performance, and age/performance relationships. Journal of Management, 29, 609-640.

United Nations (2015). World Population Ageing 2015, ST/ESA/SER.A/390, Department of Economic and Social Affairs.

Van Dalen, H. P., Henkens, K., \& Schippers, J. (2010). Productivity of older workers: Perceptions of employers and employees. Population and Development Review, 36, 309-330.

Van Ours, J. C. (2009). Will you still need me: When I'm 64? De Economist, 157, 441-460.

Van Ours, J. C., \& Stoeldraijer, L. (2011). Age, wage and productivity in Dutch manufacturing. De Economist, 159, 113-137.

Waldman, D., \& Avolio, B. J. (1986). A meta-analysis of age differences in job performance. Journal of Applied Psychology, 71, 33-38.

Williams, R. (2016). Understanding and interpreting generalized ordered logit models. The Journal of Mathematical Sociology, 40, 7-20.

Publisher's Note Springer Nature remains neutral with regard to jurisdictional claims in published maps and institutional affiliations. 